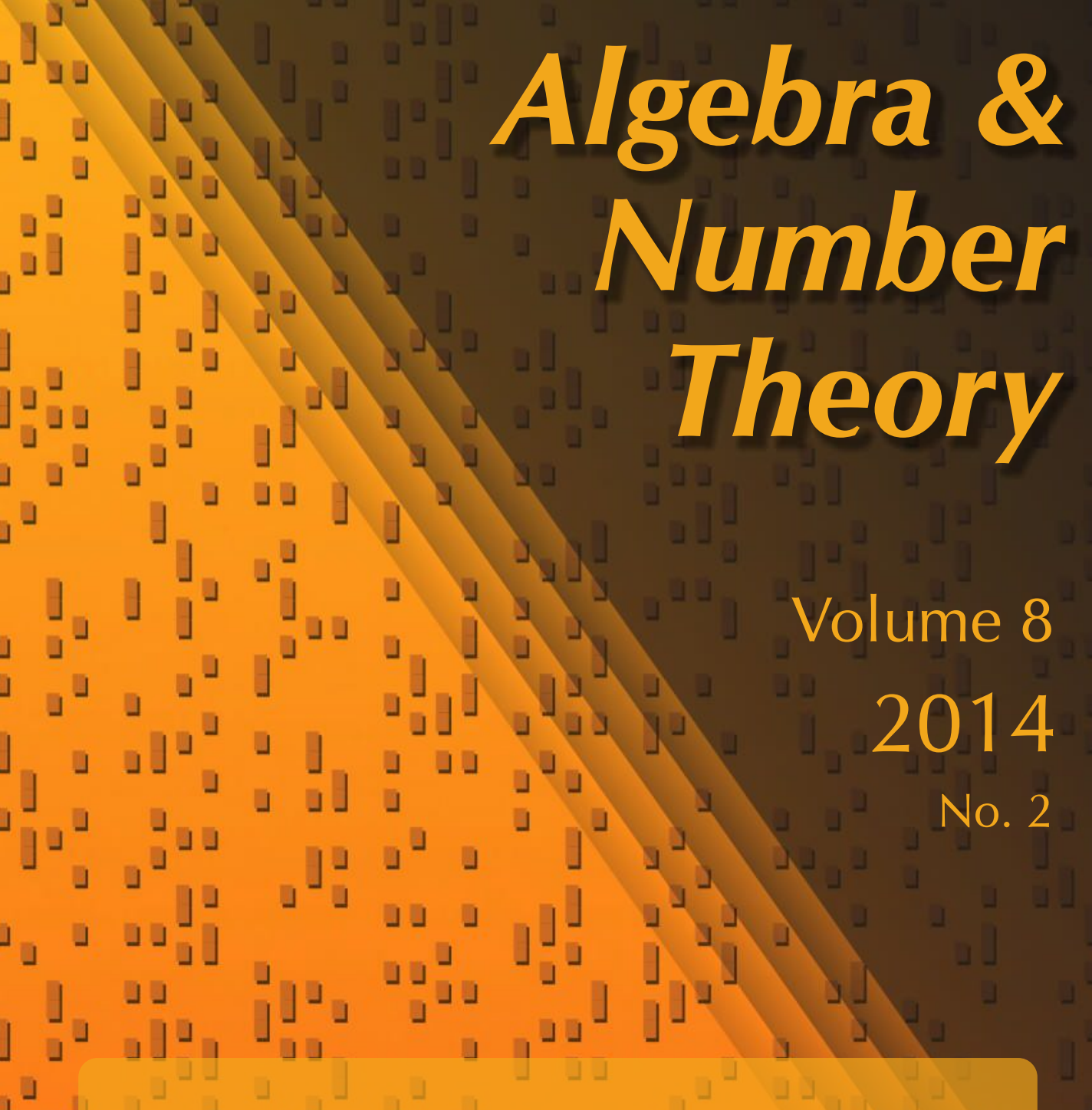

Groups with exactly one irreducible character of

degree divisible by $p$

\lrcorner

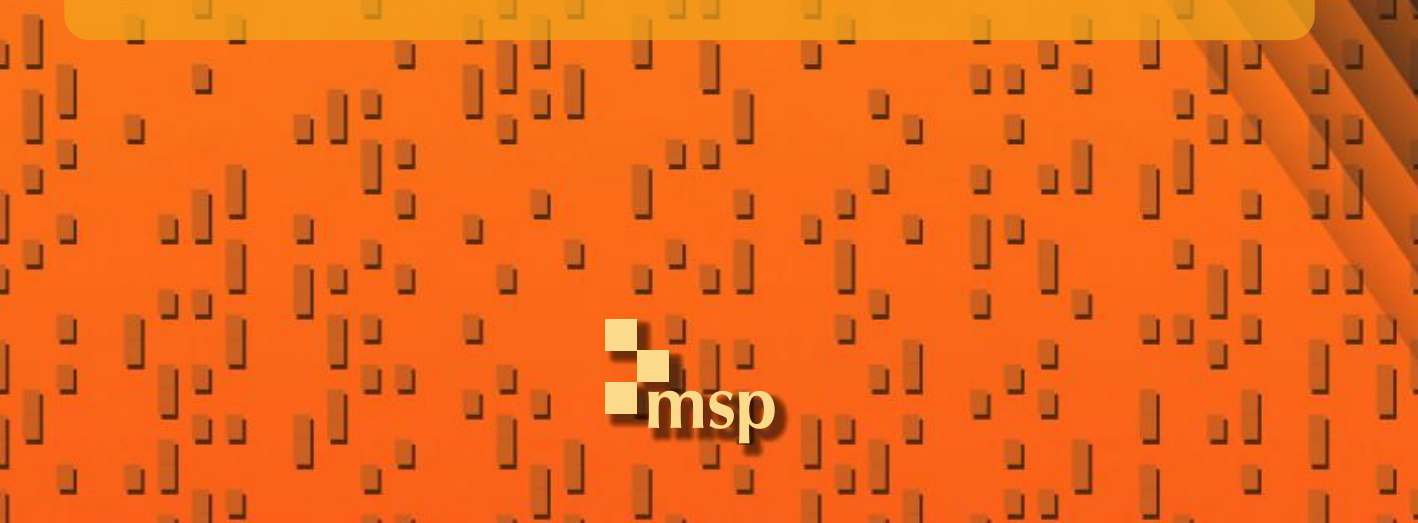




\title{
Groups with exactly one irreducible character of degree divisible by $p$
}

\author{
Daniel Goldstein, Robert M. Guralnick, Mark L. Lewis, \\ Alexander Moretó, Gabriel Navarro and Pham Huu Tiep
}

Let $p$ be a prime. We characterize those finite groups which have precisely one irreducible character of degree divisible by $p$.

Minimal situations constitute a classical theme in group theory. Not only do they arise naturally, but they also provide valuable hints in searching for general patterns. In this paper, we are concerned with character degrees. One of the key results on character degrees is the Itô-Michler theorem, which asserts that a prime $p$ does not divide the degree of any complex irreducible character of a finite group $G$ if and only if $G$ has a normal, abelian Sylow p-subgroup. In [Isaacs et al. 2009], Isaacs together with the fourth, fifth, and sixth authors of this paper studied the finite groups that have only one character degree divisible by $p$. They proved, among other things, that the Sylow $p$-subgroups of those groups were metabelian. This suggested that the derived length of the Sylow $p$-subgroups might be related with the number of different character degrees divisible by $p$. However, nothing could be said in [Isaacs et al. 2009] on how large $p$-Sylow normalizers were inside $G$. (As a trivial example, the dihedral group of order $2 n$ for $n$ odd has a unique character degree divisible by 2, and a self-normalizing Sylow 2-subgroup of order 2.)

In this paper, we go further and completely classify the finite groups with exactly one irreducible character of degree divisible by $p$. Our focus now therefore is not only on the set of character degrees but also on the multiplicity of the number of irreducible characters of each degree. In Section 1, we define the terms semiextraspecial, ultraspecial, and doubly transitive Frobenius groups of Dickson type.

We would like to thank the referee for the helpful suggestions. Guralnick and Tiep gratefully acknowledge the support of the NSF (grants DMS-1001962, DMS-0901241, and DMS-1201374). Guralnick was also partially supported by the Simons Foundation fellowship 224965. The research of Moretó and Navarro is partially supported by the Spanish Ministerio de Educación y Ciencia proyecto MTM2010-15296, and Prometeo/Generalitat Valenciana.

MSC2010: 20C15.

Keywords: finite groups, characters, character degrees. 
Main theorem. Let $p$ be a prime, and let $G$ be a finite group. Then $G$ has exactly one irreducible character of degree divisible by $p$ if and only if one of the following statements holds:

(i) $p=2$ and $G$ is an extraspecial 2-group.

(ii) $p=2$ and $G=\mathrm{S}_{4}$.

(iii) $p=3$ and $G$ is the semidirect product of $\mathrm{SL}_{2}(3)$ acting on its natural module $\left(\mathbb{F}_{3}\right)^{2}$.

(iv) $G$ is a doubly transitive Frobenius group whose Frobenius complement has a nontrivial cyclic normal Sylow p-subgroup.

(v) $p$ is odd and $G=H \ltimes K$, where $K=F(G)$ is an ultraspecial q-group for some prime $q \neq p, H$ has a normal cyclic Sylow p-subgroup $P, P$ acts trivially on $K^{\prime}$ and $G / K^{\prime}$ is a doubly transitive Frobenius group of Dickson type.

(vi) $G=H P$, where $P$ is a normal semi-extraspecial Sylow p-subgroup and $H$ is a group of order $\left|P^{\prime}\right|-1$ so that $H P^{\prime}$ is a doubly transitive Frobenius group.

(vii) Either $G$ is $\mathrm{PSL}_{2}(q)$ or $\mathrm{SL}_{2}(q)$ or there exists a minimal normal elementary abelian p-subgroup $V$ of order $q^{2}$ in $G$ so that $G / V=\mathrm{SL}_{2}(q)$ and $V$ can be viewed as a 2-dimensional irreducible module of $G / V$ over $\operatorname{End}_{G / V}(V) \cong \mathbb{F}_{q^{2}}$, where $q=p^{a} \geq 4$ is a power of $p$.

(viii) $p=3$ and $G=\mathrm{S}_{5}$.

(ix) $p=3$ and $G=M_{11}$.

Inspecting the groups listed in the main theorem, we see that either these groups have normal Sylow $p$-subgroups or their Sylow normalizers are maximal subgroups. Thus, as a corollary of the main theorem, we obtain:

Corollary. Suppose that $G$ is a finite group with exactly one irreducible character of degree divisible by $p$. Let $P \in \operatorname{Syl}_{p}(G)$. If $P$ is not normal in $G$, then $\mathbf{N}_{G}(P)$ is maximal in $G$.

This corollary suggests that, perhaps, the number of irreducible characters of $G$ of degree divisible by $p$ is bounded by the length of any saturated chain of subgroups between $\mathbf{N}_{G}(P)$ and $G$.

We now mention a connection of this problem with block theory and Brauer's height zero conjecture. We suppose that $G$ is a group and that $G$ has only one irreducible character whose degree is divisible by $p$. If $G$ has more than one block, then there must exist at least one block where all the characters have height zero and this block will have maximal defect; in particular, take any of the blocks not containing the character whose degree is divisible by $p$. Since such a block has maximal defect, its defect group will be a Sylow $p$-subgroup of $G$, and then Brauer's height zero conjecture, if true, would imply that the Sylow $p$-subgroup must be 
abelian. Thus, in light of Brauer's height zero conjecture, we would expect that either our group would have abelian Sylow $p$-subgroups or that the principal block is the only $p$-block.

By the Gluck-Wolf theorem (Theorem 12.10 of [Manz and Wolf 1993]), we know that Brauer's height zero conjecture is true for $p$-solvable groups. Thus, it is possible that the Gluck-Wolf theorem might give a different approach to proving our result for $p$-solvable groups (but probably not shorter). In particular, if $G$ does not have a single $p$-block, then we know that $G$ must have abelian Sylow $p$-subgroups. If $G$ does have a single $p$-block, then $\mathbf{O}_{p^{\prime}}(G)=1$, and so $\mathbf{O}_{p}(G)>1$. In particular, if $\mathbf{O}_{p}(G)$ is not abelian, then all the nonlinear irreducible characters in $\operatorname{Irr}\left(\mathbf{O}_{p}(G)\right)$ must be conjugate in $G$. On the other hand, by Theorem A of [Isaacs et al. 2009], we know that a $p$-solvable group $G$ having only one irreducible character degree divisible by $p$ and $\mathbf{O}_{p}(G)$ nonabelian must have that $\mathbf{O}_{p}(G)$ is a Sylow $p$-subgroup. Thus, our theorem could be viewed as classifying those groups $G$ having a normal Sylow $p$-subgroup $P$ where all the nonlinear irreducible characters of $P$ are $G$-conjugate.

This suggests that it might be worth studying the following problem which looks to us to be difficult: classify the pairs $(G, N)$ with $N$ normal in $G$ such that all the characters of $N$ with degree divisible by $p$ are conjugate in $G$. A closely related problem would be to classify the groups $G$ where all the irreducible characters of $G$ with degree divisible by $p$ are Galois conjugate. While we hesitate to predict what such a classification would look like, we observe that an extraspecial $p$-group for any prime $p$ will be an example. We expect that the $p$-solvable examples that are not nilpotent will involve Frobenius groups and a careful analysis of the conjugacy of elements of order $p$ as in our examples. For the non- $p$-solvable groups, one would begin by looking at the simple groups having the property that all irreducible characters whose degrees are divisible by $p$ are conjugate (under outer automorphisms or under Galois automorphisms). Using the classification of finite simple groups, one can show that the only nonabelian simple groups with this property are $\operatorname{PSL}_{2}(q), J_{1}$, and $M_{11}$ (see Corollary 7.5 of [Isaacs et al. 2009]). In fact, [Isaacs et al. 2009] studied a somewhat more general condition. The stronger condition that all nonlinear irreducible characters of the same degree are Galois conjugate was treated in the recent paper [Dolfi et al. 2013].

Our paper is structured as follows: in Section 1, we classify the $p$-solvable groups with exactly one irreducible character of degree divisible by $p$. The classification is first proved under the additional hypothesis that the group is solvable. We then produce examples of solvable groups that satisfy conclusion (vi) of the classification. The classification is then proved under the hypothesis that the group is $p$-solvable, but not solvable. This case is split depending on whether the Sylow $p$-subgroup is abelian or nonabelian. We also find some additional constraints when the group 
is not solvable, but it is $p$-solvable and has a nonabelian Sylow $p$-subgroup. We construct $p$-solvable groups that are not solvable and satisfy conclusion (vi) of the classification. Finally, in Section 2 we take care of the classification of the non- $p$-solvable groups.

\section{1. $p$-solvable groups}

In this section we state the classification for $p$-solvable groups. In Section 2 of [Dolfi et al. 2009], the reader can find definitions of the relevant terms below. Recall that $P$ is a semi-extraspecial $p$-group if $P / N$ is an extraspecial $p$-group for every subgroup $N$ in $\mathbf{Z}(P)$ with $|\mathbf{Z}(P): N|=p$. It is known that if $P$ is a semi-extraspecial $p$-group, then $P^{\prime}=\mathbf{Z}(P)=\Phi(P)$ and $\mathbf{Z}(P)$ is elementary abelian. Also, $\left|P^{\prime}\right|^{2} \leq\left|P: P^{\prime}\right|$. For details, see [Fernández-Alcober and Moretó 2001], for instance. A group $P$ is ultraspecial if $P$ is a semi-extraspecial $p$-group that satisfies $\left|P^{\prime}\right|^{2}=\left|P: P^{\prime}\right|$. In this section, we prove the following result, which is the $p$-solvable portion of the main theorem.

Theorem 1.1. Fix a prime number $p$, and let $G$ be a finite p-solvable group. Then $G$ has exactly one irreducible character of degree divisible by $p$ if and only if one of the following holds:

(i) $p=2$ and $G$ is an extraspecial 2-group.

(ii) $p=2$ and $G=\mathrm{S}_{4}$.

(iii) $p=3$ and $G$ is the semidirect product of $\mathrm{SL}_{2}(3)$ acting on its natural module.

(iv) $G$ is a doubly transitive Frobenius group whose Frobenius complement has a nontrivial cyclic normal Sylow p-subgroup.

(v) $p$ is odd and $G=H \ltimes K$ where $K=F(G)$ is an ultraspecial q-group for some prime $q \neq p, H$ has a normal cyclic Sylow $p$-subgroup $P, P$ acts trivially on $K^{\prime}$ and $G / K^{\prime}$ is a doubly transitive Frobenius group of Dickson type.

(vi) $G=H P$, where $P$ is a normal semi-extraspecial Sylow p-subgroup and $H$ is a group of order $\left|P^{\prime}\right|-1$ so that $H P^{\prime}$ is a doubly transitive Frobenius group.

Clearly, the groups in conclusions (i), (ii), and (iii) exist and are solvable. Doubly transitive Frobenius groups have been studied in a number of different places. As mentioned in [Dolfi et al. 2009], the doubly transitive Frobenius groups are in bijection with the finite near-rings. Most near-rings are obtained by Galois twists of finite fields and these are said to be of Dickson type. There are also seven near-rings that are said to be of exceptional type. The doubly transitive Frobenius groups of Dickson type are solvable. Four of the doubly transitive Frobenius groups of exceptional type are solvable and the other three are nonsolvable. As mentioned in [Dolfi et al. 2009], one of the solvable and two of the nonsolvable doubly transitive 
groups of exceptional type have a nontrivial cyclic normal Sylow $p$-subgroup. Thus, there are two nonsolvable groups that satisfy conclusion (iv).

In conclusion (v), since $G / K^{\prime}$ is doubly transitive of Dickson type and $K$ is a $q$-group for some prime, we see that $G$ must be solvable. We will see that the groups in (v) have exactly one conjugacy class whose size is divisible by $p$. In Section 5 of [Dolfi et al. 2009], they construct groups that satisfy conclusion (v), and they give conditions on when such groups can be constructed.

Groups that satisfy conclusion (vi) can be found on page 383 of [Gagola 1983]. Using this construction, one can find an example with $\left|P^{\prime}\right|=p^{a}$ for every prime $p$ and integer $a \geq 1$. We will use a variation on this construction to find groups that satisfy conclusion (vi) where $H P^{\prime}$ is any doubly transitive Frobenius group of Dickson type. We will also present a variation on this construction to produce an example where $P$ is not ultraspecial.

We will also show that we can find groups that satisfy conclusion (vi) where $H P^{\prime}$ is any of the exceptional doubly transitive Frobenius groups. We will also find additional restrictions on the groups arising in this case. (See Theorem 1.20, where we essentially classify such groups. It is perhaps remarkable how large the exponent of $\left|P / P^{\prime}\right|$ needs to be in any of these groups.)

We claim that it is easy to see that if $G$ is one of the groups in (i)-(vi), then it has exactly one irreducible character of degree divisible by $p$. Thus, we will work to prove that if $G$ is $p$-solvable and has exactly one irreducible character of degree divisible by $p$, then $G$ is one of the groups in (i)-(vi).

Preliminaries. In this section, we present several results from other sources.

Lemma 1.2 [Lewis 2001, Lemma 1]. Suppose a solvable group G acts faithfully on a group $V$, and let $p$ be a prime divisor of $|G|$. Assume for each nonidentity element $v \in V$ that $\mathbf{C}_{G}(v)$ contains a unique Sylow p-subgroup of $G$. Then $G$ is a subgroup of the semilinear group on $V$ or $p=3,|V|=9$ and $G$ is one of the groups $\mathrm{SL}_{2}$ (3) or $\mathrm{GL}_{2}(3)$.

This next result was proved by Noritzsch. We write $\operatorname{cd}(G)=\{\chi(1) \mid \chi \in \operatorname{Irr}(G)\}$ for the set of irreducible character degrees of $G$.

Lemma 1.3 [Noritzsch 1995, Lemma 1.10]. Let $V<N<G$ be normal subgroups of a finite group $G$ such that $G / N$ and $N / V$ are cyclic of order $a$ and $b$, respectively. Moreover, let $V$ be elementary abelian and suppose that both $G / V$ and $N$ are Frobenius groups with kernel $N / V$ and $V$, respectively. Then

$$
\operatorname{cd}(G) \cup\{a b\}=\{1, a\} \cup\{i b \mid i \text { divides } a\} .
$$

For nilpotent groups, the result is immediate. (This is essentially proved in [Seitz 1968], but we have decided to include our own short proof.) 
Lemma 1.4. Let $G$ be a nilpotent group, and let $p$ be a prime. Then $G$ has exactly one irreducible character of degree divisible by $p$ if and only if $G$ is an extraspecial 2-group.

Proof. Obviously $G$ must be a nonabelian $p$-group. Let $Z=\mathbf{Z}(G) \cap G^{\prime}$, and note that $Z>1$. If $\alpha$ and $\beta$ are distinct nontrivial characters of $Z$, then any irreducible constituent of $\alpha^{G}$ or $\beta^{G}$ has degree a nontrivial power of $p$ and clearly $\alpha^{G}$ and $\beta^{G}$ have no common constituents. Thus, $Z$ has only one nontrivial character, whence $|Z|=2$ and so $G$ is a 2-group. We see that $G / Z$ has no irreducible characters of degree bigger than 1 , and hence, $Z=G^{\prime}$. Thus, $G$ has $|G| / 2$ distinct linear characters and so the nonlinear irreducible character has degree $[G: Z]^{1 / 2}$, whence $Z=\mathbf{Z}(G)$. Thus, $G$ is an extraspecial 2-group.

Groups with exactly one conjugacy class with size divisible by a prime $p$ have been classified in [Dolfi et al. 2009]. Next is the classification.

Lemma 1.5 [Dolfi et al. 2009, Theorem A]. Let $G$ be a finite group and $p$ a prime. Then $G$ has exactly one conjugacy class of size divisible by $p$ if and only if $G$ is one of the following groups:

(i) $G$ is a Frobenius group with Frobenius complement of order 2 and Frobenius kernel of order divisible by $p$.

(ii) $G$ is a doubly transitive Frobenius group whose Frobenius complement has a nontrivial central Sylow p-subgroup.

(iii) $p$ is odd, $G=K H$, where $K=F(G)$ is an ultraspecial q-group, $q$ prime, $H=\mathbf{C}_{G}(P)$ for a Sylow p-subgroup $P$ of $G, K \cap H=\mathbf{Z}(K)$ and $G / \mathbf{Z}(K)$ is a doubly transitive Frobenius group of Dickson type.

Given an element $g \in G$, we write $\operatorname{cl}_{G}(g)$ for the conjugacy class of $g$ in $G$.

Lemma 1.6. Let $p$ be a prime number. Assume that $G$ has a normal p-complement and that $G$ is not nilpotent. If $G$ has exactly one irreducible character of degree divisible by $p$, then it has exactly one conjugacy class of size divisible by $p$.

Proof. Write $G=A N$, where $A$ is a Sylow $p$-subgroup of $G$ and $N$ is a normal $p$ complement. Assume first that $A$ is not abelian. By Lemma 1.4, $A$ is an extraspecial 2-group. Furthermore, by hypothesis every irreducible character of $N$ is $A$-invariant. We deduce that $N=1$. This contradiction implies that $A$ is abelian.

By hypothesis, there exists a unique $A$-orbit of irreducible characters of $N$ that are not $A$-invariant. Let $\theta \in \operatorname{Irr}(N)$ be a character that is not $A$-invariant. Since $\theta$ extends to its inertia subgroup in $G$ and our hypothesis implies that there exists a unique irreducible character of $G$ lying over $\theta$, we have that $G_{\theta}=N$. In other words, the action of $A$ on $\operatorname{Irr}(N)$ has exactly one regular orbit, and all other orbits have size one. Since the actions on characters and classes are permutation isomorphic, the 
same happens for the action of $A$ on the set of conjugacy classes of $N$. We deduce that there exists a unique $G$-conjugacy class contained in $N$ of size a multiple of $p$.

Now, consider an element $g \in G \backslash N$. We must prove that $p$ does not divide $\left|\mathrm{cl}_{G}(g)\right|$. Since $N$ is a normal Hall $p^{\prime}$-subgroup, $p$ divides $o(g)$. If $o(g)$ is a power of $p$, then $g$ belongs to some Sylow $p$-subgroup of $G$. Since the Sylow $p$-subgroups of $G$ are abelian, it follows that $\left|\mathrm{cl}_{G}(g)\right|$ is a $p^{\prime}$-number, as desired. Hence, we may assume that the order of $g$ is not a prime power, and we can write $g=g_{p} g_{p^{\prime}}$ as the product of its $p$-part and its $p^{\prime}$-part, where $g_{p} \neq 1$ and $g_{p^{\prime}} \neq 1$. Observe that $g_{p^{\prime}}$ belongs to $N$ and commutes with the nontrivial $p$-element $g_{p}$. By the previous paragraph, $g_{p^{\prime}}$ commutes with a Sylow $p$-subgroup $T$ that contains $g_{p}$. It follows that $g$ commutes with $T$, and hence, $\left|\mathrm{cl}_{G}(g)\right|$ is a $p^{\prime}$-number, as desired.

Looking at the conclusion of Lemma 1.5, it is not difficult to see that only the groups in conclusions (ii) and (iii) have exactly one irreducible character whose degree is divisible by $p$, and that these groups satisfy conclusions (iv) and (v) of Theorem 1.1. Thus, combining Lemma 1.6 with Lemma 1.5 yields the following corollary.

Corollary 1.7. Let $p$ be a prime number. Assume G has a normal p-complement and $G$ is not nilpotent. If $G$ has exactly one irreducible character of degree divisible by $p$, then $G$ is one of the groups in (iv) or (v) of Theorem 1.1.

Let $K$ be a normal subgroup of $G$. We use $\operatorname{Irr}(G \mid K)$ to denote the characters in $\operatorname{Irr}(G)$ that do not contain $K$ in their kernels.

Lemma 1.8. Let $p$ be a prime number. Assume that $G$ has a normal Sylow psubgroup but that $G$ is not a p-group. If $G$ has exactly one irreducible character of degree divisible by $p$, then $G$ is one of the groups in (vi) of Theorem 1.1.

Proof. Take $P$ to be the Sylow $p$-subgroup of $G$, and let $H$ be a Hall $p$-complement for $G$. Observe that $p$ divides the degree of every character in $\operatorname{Irr}\left(G \mid P^{\prime}\right)$. This implies that $\operatorname{Irr}\left(G \mid P^{\prime}\right)$ contains a unique character $\chi$. It is not difficult to see that $\chi$ vanishes on $G \backslash P^{\prime}$. Also, $G$ has a unique orbit on the nonprincipal characters of $\operatorname{Irr}\left(P^{\prime}\right)$. By Theorem 6.32 of [Isaacs 1976], this implies that $P^{\prime}$ contains only one nonidentity conjugacy class of $G$. This implies $\chi$ is one of the characters studied by Gagola [1983]. From Lemma 2.1 of [Gagola 1983], we see that $P^{\prime} H$ is a doubly transitive Frobenius group. We deduce that $P^{\prime}$ is a minimal normal in $G$, and $P^{\prime}$ is central in $P$. In the language of [Chillag and Macdonald 1984], $\left(G, P^{\prime}\right)$ is a Camina pair, and using Lemma 4.2 from that paper and the fact that $P^{\prime}$ is central in $P$, one sees that $P$ is semi-extraspecial.

The solvable case. We now prove Theorem 1.1 under the addition hypothesis that $G$ is solvable. 
Theorem 1.9. Let $p$ be a prime, and let $G$ be a group having exactly one irreducible character whose degree is divisible by $p$. If $G$ is solvable, then $G$ satisfies conclusions (i)-(vi) of Theorem 1.1.

Proof. Assume that $G$ has exactly one irreducible character of degree divisible by $p$. We want to prove that $G$ is one of the groups in (i)-(vi). Let $G$ be a minimal counterexample.

Step 1: If $\mathbf{O}_{p}(G)>1$, then $\mathbf{O}_{p}(G)$ is a Sylow subgroup of $G$.

Write $V=\mathbf{O}_{p}(G)$, and assume that $V$ is not a Sylow $p$-subgroup of $G$. Now, $G / V$ has a nontrivial, nonnormal Sylow $p$-subgroup, so $p$ must divide the degree of some character in $\operatorname{Irr}(G / V)$ by Itô's theorem (Theorem 12.33 of [Isaacs 1976]). Hence, $G / V$ will have exactly one irreducible character of degree divisible by $p$, and thus, $G / V$ is not a counterexample. Since $G / V$ does not have a nontrivial normal $p$-subgroup, $G / V$ is one of the groups in (iv) or (v) of Theorem 1.1.

Substep $1 a$ : $V$ is a minimal normal subgroup of $G$.

Let $K$ be a normal subgroup of $G$ such that $V / K$ is a chief factor of $G$. Assume that $K>1$. Since $G / K$ is not a counterexample, $G / K=\mathrm{S}_{4}$ and $p=2$ or $G / K$ is the group in (vi) and $p=3$. In both cases, a Sylow $p$-subgroup is not abelian, so we may apply Theorem 12.9 of [Manz and Wolf 1993] to see that $\operatorname{Irr}(G \mid K)$ must contain a character of degree divisible by $p$. Since $\operatorname{Irr}(G / K)$ already contains a character of degree divisible by $p$, this is a contradiction. Therefore, we must have $K=1$, and $V$ is a minimal normal subgroup of $G$.

Substep $1 b: V=F(G)$.

Assume that $V<F=F(G)$. Let $E / V=F(G / V)$. Suppose first that $F=E$. Since $G / V$ satisfies conclusion (iv) or (v), we know that $F=V \times W$, where $W$ is a $q$-group for some prime $q \neq p$, and $G / V W^{\prime}$ is a doubly transitive Frobenius group. This implies that $W / W^{\prime}$ is an elementary abelian $q$-group and $\left(1_{V} \times \lambda\right)^{G} \in \operatorname{Irr}(G)$ for any character $1_{W} \neq \lambda \in \operatorname{Irr}\left(W / W^{\prime}\right)$. It is not difficult to show that $(\alpha \times \lambda)^{G} \in \operatorname{Irr}(G)$ for every character $\alpha \in \operatorname{Irr}(V)$. Since $1_{V} \times \lambda$ will be in a different $G$-orbit than $\alpha \times \lambda$ when $\alpha \neq 1_{V}$, we deduce that $G$ has more than one irreducible character of degree divisible by $p$, a contradiction. Thus, we may assume that $F<E$.

Recall that $G / V$ satisfies either conclusion (iv) or conclusion (v). In both cases, we know that $G / E^{\prime} V$ is a doubly transitive Frobenius group, so $E / E^{\prime} V$ is a chief factor for $G$. If $G / V$ satisfies conclusion (iv), then $E / V$ is abelian, so $E / V$ is a chief factor, and we cannot have $F<V<E$. Thus, the claim is proved in this case, and we may assume that $G / V$ satisfies conclusion (v).

We know that $E / V$ is a semi-extraspecial group, so every normal subgroup of $E / V$ either contains $E^{\prime} V / V$ or is contained in $E^{\prime} V / V$ (by Corollary 8.3 of [Fernández-Alcober and Moretó 2001]). Since $E / E^{\prime} V$ is a chief factor and $F<E$, this implies that $F \leq E^{\prime} V$. As $V<F$, we still have $F=V \times W$ where $W>1$ is a 
normal $q$-group in $G$. Also, because $G / E^{\prime} V$ is a doubly transitive Frobenius group, we know that $G / E^{\prime} V$ does not have a normal Sylow $p$-subgroup. Hence, $G / W$ does not have a normal Sylow $p$-subgroup. By Itô's theorem, this implies that $p$ divides the degree of some character in $\operatorname{Irr}(G / W)$. Now, $G / W$ is not a counterexample to the theorem and it has a nontrivial normal $p$-subgroup, and a Sylow $p$-subgroup that is not normal. It follows that $G / W$ satisfies either conclusion (ii) or conclusion (vi), and both of these have a nonabelian Sylow $p$-subgroup. Thus, we may apply Theorem 12.9 of [Manz and Wolf 1993] to see that $\operatorname{Irr}(G \mid W)$ has a character with degree divisible by $p$, a contradiction. This completes the proof of the claim that $V=F(G)$.

\section{Substep 1c: Proof of step 1.}

Now, $G / V$ acts faithfully on $V$, and $\mathbf{C}_{G}(v)$ contains a unique Sylow $p$-subgroup of $G$ for any nonidentity $v \in V$. We can apply Lemma 1.2, and deduce that either $p=3, G / V=\mathrm{SL}_{2}(3)$ and $|V|=9$ or $G / V$ is a subgroup of the semilinear group on $V$. In the first case, we obtain that $G$ is the group of type (vi). This is a contradiction. In the second case, we have that $G / V$ is a metacyclic doubly transitive Frobenius group with kernel, say, $K / V$ and that the action of $K / V$ on $V$ is Frobenius. By Lemma 1.3, we deduce that $|G / K|=p$. But since $|K / V|=p+1$ is prime, we deduce that $p=2$ and $G / V=\mathrm{S}_{3}$. We claim that $|V|=4$. For every nonprincipal irreducible character $\lambda$ of $V$, the inertia subgroup of $\lambda$ in $G$ is a Sylow 2 -subgroup of $G$. Since $G$ has 3 Sylow 2 -subgroups $\left\{P_{1}, P_{2}, P_{3}\right\}$, we deduce that

$$
\operatorname{Irr}(F(G)) \backslash\left\{1_{F(G)}\right\}=\bigcup_{i=1}^{3} C_{\operatorname{Irr}(F(G))}\left(P_{i}\right),
$$

and this union is disjoint. In particular, $|V|-1=3 \cdot 2^{a}$ for some integer $a$. Since $V$ is a 2-group and $|V|=3 \cdot 2^{a}+1$, we deduce that $a=0$ and $|V|=4$. This implies that $G=\mathrm{S}_{4}$, again a contradiction. This means that if $V>1$, then $V$ is a Sylow $p$-subgroup, proving step 1.

Let $H$ be a Hall p-complement of $G$.

Step 2: $\mathbf{O}_{p}(G)=1$.

Suppose $\mathbf{O}_{p}(G)>1$. By step $1, G$ has a normal Sylow $p$-subgroup. By Lemma 1.8, $G$ satisfies conclusion (vi) and this contradicts the choice of $G$ as a counterexample.

Step 3: $G$ has a normal $p$-complement.

Assume that $G$ does not have a normal $p$-complement. By Theorem A of [Isaacs et al. 2009], $G$ has a cyclic Sylow $p$-subgroup. Write $X=\mathbf{O}_{p^{\prime}}(G)$ and $Y / X=\mathbf{O}_{p}(G / X)$. The group $Y / X$ is isomorphic to a Sylow $p$-subgroup of $G$ and $G / Y$ is isomorphic to a $p^{\prime}$-subgroup of $\operatorname{Aut}(Y / X)$. Hence, $G / Y$ is a cyclic 
group whose order is a divisor of $p-1$. Write $Y=P X$, where $P$ is a Sylow $p$-subgroup of $G$. Since we are assuming that $G / Y>Y / Y$, it follows that $p>2$. Set $V=[P, X]$, and let $V / W$ be a chief factor of $G$. Let $r$ be the prime so that $V / W$ is an elementary abelian $r$-group.

Substep 3a: If $W>1$, then $W$ is nilpotent, $W=V^{\prime}$, and all nonlinear irreducible characters of $V$ are $P$-invariant.

By the minimality of $G$ as a counterexample to the theorem, $G / W$ is one of the groups described in (i)-(vi). We know that $p>2$, so $G / W$ is one of the groups in (iii)-(vi). The Sylow $p$-subgroups of $G / W$ are cyclic, so it is not one of the groups of type (iii) or (vi) either. The Sylow $p$-subgroups of $G / W$ do not act trivially on the minimal normal subgroup $V / W$, so $G / W$ cannot be of type (v). It follows that $G / W$ is a doubly transitive Frobenius group whose complement has a cyclic normal Sylow $p$-subgroup. In particular, $K=F(G) \leq V$. Since $G$ has a unique irreducible character of degree divisible by $p$, it is easy to see that $V^{\prime}=W$. Also, since $p$ will not divide the degrees of any of the characters in $\operatorname{Irr}\left(G \mid V^{\prime}\right)$, all the nonlinear characters of $V$ are fixed by $P$. By Theorem A of [Isaacs 1989], $W$ is nilpotent.

Substep $3 b$ : If $W>1$, then $V$ is an $r$-group.

Assume that this is not true. Then there exists a normal subgroup $J$ of $G$ so that $W / J$ is a chief factor of $G$ which is a $t$-group, for some prime $t \neq r$. For any character $\tau \in \operatorname{Irr}(W / J)$, we see that the stabilizer $G_{\tau}$ contains a full Sylow $p$-subgroup of $G$. Also, $\tau$ extends to $P V_{\tau}$. Since the action of $P$ on $V / W$ is Frobenius, it follows from our hypothesis that $V_{\tau}=W$. Hence, the action of $V / W$ on $W / J$ is Frobenius, so $V / W$ is cyclic of order $r$ and $G / V$ is cyclic. It follows that $G$ has a normal $p$-complement, a contradiction. This proves the claim that $W$ is an $r$-group. As a consequence, $V$ is an $r$-group.

Substep 3c: $W=1$.

Suppose $W>1$. Fix a character $\theta \in \operatorname{Irr}(V \mid W)=\operatorname{Irr}\left(V \mid V^{\prime}\right)$, so $\theta$ is nonlinear. We have seen that $\theta$ must be $P$-invariant. So there exists a character $\mu \in \operatorname{Irr}(W)$ that is a constituent of $\theta_{W}$ and is $P$-invariant. By Problem 13.10 of [Isaacs 1976], there exists a unique irreducible constituent of $\mu^{V}$ that is $P$-invariant. But all the members of $\operatorname{Irr}(V \mid W)$ are $P$-invariant. It follows that $\mu^{V}=e \theta$ for some integer $e$. In particular, $\theta$ vanishes on the set $V \backslash W$. By Lemma 3.1 of [Lewis et al. 2005], we see that $V$ is a semi-extraspecial $r$-group. One can easily see that $P$ acts trivially on $W$, so $G$ is one of the groups in (v). This contradiction implies that $W=1$.

Let $C=\mathbf{C}_{G}(V)$.

Substep 3d: $G / C$ acts transitively on $V \backslash\{1\}$. 
Observe that $P V / V$ is a Sylow $p$-subgroup of $G / V$. Since $\mathbf{O}_{p}(G)=1$, we know that $P$ acts faithfully on $V$. Also, $V=\mathbf{C}_{V}(P) \times[V, P]$ by Fitting's lemma. We have that $\mathbf{C}_{V}(P)$ is normal in $G$ (since $P V$ is normal in $G$ ), and $V$ is a minimal normal subgroup of $G$. It follows that $\mathbf{C}_{V}(P)=1$, so the action of $P$ on $V$ is Frobenius (by Theorem A of [Isaacs et al. 2009]). It follows that the degree of any of the members of $\operatorname{Irr}(G \mid V)$ is a multiple of $p$. By hypothesis, $|\operatorname{Irr}(G \mid V)|=1$. This implies that the action of $G / C$ on $V \backslash\{1\}$ is transitive.

Now, we can apply Theorem 6.8 of [Manz and Wolf 1993] to determine the structure of $G / C$.

Substep $3 e: C \leq X$.

On the other hand, consider any character $\theta \in \operatorname{Irr}(X \mid V)$. Since $P$ acts Frobeniusly on $V$, we know that the stabilizer of any nonprincipal irreducible character of $V$ will be contained in $X$, and so, all the characters in $\operatorname{Irr}(X \mid V)$ induce irreducibly to $Y$. In particular, $\theta^{Y} \in \operatorname{Irr}(Y)$. But $G / Y$ is cyclic, and $\left(\theta^{Y}\right)^{G}$ has a unique irreducible constituent. Clifford theory implies that $\theta^{G} \in \operatorname{Irr}(G)$. In other words, any member of $\operatorname{Irr}(X \mid V)$ induces irreducibly to the same character of $G$. Also, the action of $P$ on $V$ is Frobenius, so $C$ is a $p^{\prime}$-group. This implies that $C \leq X$.

Substep 3f: $G / C$ must be one the "exceptional groups" in the conclusion of Theorem 6.8 of [Manz and Wolf 1993].

Suppose first that $G / C$ is isomorphic to a subgroup of the semilinear group of $V$. This implies that $G / C$ is metacyclic. Write $F / C=F(G / C)$, and note that $G / F$ is cyclic. Put $U=F \cap X \unlhd G$. Arguing as in the previous paragraph, any member of $\operatorname{Irr}(U \mid V)$ induces irreducibly to the same character of $G$. This fact and Clifford's correspondence imply for every character $\lambda \in \operatorname{Irr}(V) \backslash\left\{1_{V}\right\}$ that $\left|\operatorname{Irr}\left(U_{\lambda} \mid \lambda\right)\right|=1$. Hence, $\lambda$ is fully ramified with respect to $U_{\lambda} / V$. We deduce that $U_{\lambda}$ is a $q$-group for some prime $q$. Since the Sylow $q$-subgroups of $\Gamma(V)$ are cyclic, it follows that $U_{\lambda} / C$ is cyclic and is contained in the cyclic group $\mathbf{O}_{q}(G / C)$. In particular, $U_{\lambda} \unlhd G$. Since $G$ is transitive on $V \backslash\{1\}$, we deduce that $U_{\lambda}=C$. Because $C / V$ is fully ramified, we obtain $Z(C) \leq V$. But $V$ is minimal normal in $G$, so $Z(C)=V$.

We claim that $C=V$. Assume that this is not true. Thus, $C^{\prime} \cap V>1$, so $V \leq C^{\prime}$. But we know that $P$ acts trivially on $C / C^{\prime}$. Hence, it acts trivially on $C$ and this is a contradiction. This implies that $C=V$, and the action of $G / V$ on $V$ is Frobenius.

This proves that $G$ is a double transitive Frobenius group whose complement has a cyclic normal Sylow $p$-subgroup; that is, $G$ is of type (iv). This is a contradiction. By Theorem 6.8 of [Manz and Wolf 1993], $G / C$ is one of the exceptional groups in that theorem.

Substep 3g: Proof of step 3.

The group $G / C$ cannot be one of the groups in conclusion (a) of Theorem 6.8 of [Manz and Wolf 1993] because $F(G / C)$ is not a nonabelian group of prime power 
order. Also, it cannot be one of the groups in conclusion (b) because $F(G / C)$ contains a noncentral cyclic Sylow $p$-subgroup. Therefore, $G$ has a normal $p$ complement.

Step 4: Final contradiction.

By Corollary 1.7, $G$ is one of the groups of type (iv) or (v). This is the final contradiction.

Solvable examples. We now construct solvable groups that appear in conclusion (vi) of Theorem 1.1. In particular, for every two-transitive Frobenius group of Dickson type $D$, we find a group $G$ satisfying conclusion (vi) of Theorem 1.1 so that $\mathbf{Z}(P) H \cong D$. We will also present an example that satisfies conclusion (vi) of Theorem 1.1 where $P$ is not ultraspecial.

We start with examples that appeared in [Gagola 1983] and in [Isaacs 2011]. Let $p$ be a prime and let $n$ be a positive integer. Write $F$ for the finite field of order $p^{n}$. Take $K$ to be the matrix group

$$
\left\{\left(\begin{array}{lll}
1 & a & b \\
0 & 1 & c \\
0 & 0 & d
\end{array}\right): a, b, c \in F ; d \in F^{*}\right\} .
$$

Set

$$
P=\left\{\left(\begin{array}{lll}
1 & a & b \\
0 & 1 & c \\
0 & 0 & 1
\end{array}\right): a, b, c \in F\right\} .
$$

It is shown in [Gagola 1983] that $P$ is a normal Sylow $p$-subgroup of $K$. It is well known that $P$ is an ultraspecial group of order $p^{3 n}$. Set

$$
L=\left\{\left(\begin{array}{lll}
1 & 0 & 0 \\
0 & 1 & 0 \\
0 & 0 & d
\end{array}\right): d \in F^{*}\right\} .
$$

Observe that $L$ is a Hall $p$-complement of $K$. Also, $L$ is cyclic. It is not hard to see that $L$ acts Frobeniusly on

$$
\mathbf{Z}(P)=\left\{\left(\begin{array}{lll}
1 & 0 & b \\
0 & 1 & 0 \\
0 & 0 & 1
\end{array}\right): b \in F\right\},
$$

but that $L$ does not act Frobeniusly on $P$. We should note that in fact $K$ satisfies conclusion (vi) of Theorem 1.1. However, we need to produce more complicated examples. To do this, let $\mathscr{G}$ be the Galois group for $F$ over $Z_{p}$. We can define an action $\mathscr{G}$ on $K$ as follows: if $\sigma \in \mathscr{G}$, then $\sigma$ acts on a typical element of $K$ by acting on each of the entries of $K$. Notice that $P$ and $L$ are invariant under the action 
of $\mathscr{G}$. Also, note that the semidirect product of $\mathscr{G}$ acting on $L$ is isomorphic to the affine group on $F$. (See page 38 of [Manz and Wolf 1993].) We take $\Gamma$ to be the semidirect product of $\mathscr{G}$ acting on $K$. (We note that $\mathbf{Z}(P) L G$ is isomorphic to the affine semilinear group on $F$ which is also defined on page 38 of [Manz and Wolf 1993].)

Suppose $D=N H^{*}$ is a two-transitive Frobenius group of Dickson type of order $p^{n}\left(p^{n}-1\right)$, where $N$ is the Frobenius kernel and $H^{*}$ is the Frobenius complement. It is well known that $H^{*}$ can be embedded in the affine group of $F$ and that $N H^{*}$ is isomorphic to a subgroup of the semilinear affine group of $F$. Thus, $H^{*}$ is isomorphic to $H \subseteq L^{G}$ and $N H$ is isomorphic to $\mathbf{Z}(P) H$. We set $G=P H$, and it is not difficult to see that $G$ is the desired group.

To find an example of a group satisfying conclusion (vi) of Theorem 1.1 where $P$ is not ultraspecial, we take $\Gamma$ as above, but specialize $p=2$ and $n=3$. This implies that $|P|=2^{9}$ and $|\mathscr{G}|=3$. We define $G^{*}=P \mathscr{G}$. Observe that $\mathscr{G}$ centralizes a subgroup $Z$ of $Z(P)$ having order 2. (The fixed field under the Galois group has order 2.) It follows that $Z$ is in the center of $G^{*}$. Let $G=G^{*} / Z$. Since $P / Z$ is a nonabelian quotient of a semi-extraspecial group, it is semi-extraspecial. Since $|P: \mathbf{Z}(P)|=2^{6}$ and $|\mathbf{Z}(P): Z|=2^{2}$, it is not ultraspecial. Observe that $\mathscr{G}$ acts Frobeniusly on $\mathbf{Z}(P) / Z$, so $\mathbf{Z}(P) \mathscr{G} / Z$ is a doubly transitive Frobenius group. It follows from Lemma 2.2 of [Chillag and Macdonald 1984] that $G$ satisfies conclusion (vi) of Theorem 1.1, and this yields our example with the normal Sylow subgroup not being ultraspecial.

We will construct examples where $D \cong \mathbf{Z}(P) H$ when $D$ is a two-transitive solvable Frobenius of exceptional group later. The technique for constructing groups that satisfy conclusion (vi) of Theorem 1.1 when $\mathbf{Z}(P) H$ is exceptional is the same for solvable and nonsolvable groups. Thus, we hold off on that construction until after we handle the proof of Theorem 1.1 in the case where the group is $p$-solvable, but not solvable.

Abelian Sylow p-subgroup. We now prove Theorem 1.1 in the case that $G$ is $p$-solvable but not solvable and the Sylow $p$-subgroup of $G$ is abelian.

The following result is the only one in this section that uses the classification of finite simple groups.

Lemma 1.10. Let $p$ be prime. Let $S$ be a finite nonabelian almost-simple group not divisible by $p$ and A a nontrivial p-group of automorphisms of $S$. Then A is cyclic. Moreover, A has at least two nontrivial orbits on the irreducible characters of $S$.

Proof. It follows by the classification of finite simple groups and their automorphism groups that $F^{*}(S)$ is a finite group of Lie type and $A$ is a cyclic group of field automorphisms. By Brauer's lemma, it suffices to prove the same statement for 
conjugacy classes. We can appeal to [Dolfi et al. 2009, Lemma 3.1] to complete the proof.

We apply the previous lemma to obtain the following.

Lemma 1.11. Let $G$ be a finite group with a minimal normal subgroup $N$ that is a direct product of $t$ copies of a nonabelian finite simple group. Assume that $\mathbf{C}_{G}(N)=1$ and that either $t>1$ or that there is a prime $p$ dividing $|G|$ that does not divide $|N|$. Then $G$ has at least 2 nontrivial orbits on conjugacy classes of $N$ of size a multiple of $p$ and on irreducible characters of $N$ of degree divisible by $p$.

Proof. If $p$ divides $|N|$ and $t>1$, the result is clear (indeed, there will be at least $t$ such orbits).

If $t=1$ and $p$ does not divide $|N|$, then Lemma 1.10 applies.

We now prove Theorem 1.1 under the hypothesis that $G$ is $p$-solvable but not solvable and that $G$ has an abelian Sylow $p$-subgroup.

Theorem 1.12. Let $p$ be a prime, and let $G$ be a group having exactly one irreducible character whose degree is divisible by $p$. Suppose $G$ is p-solvable, but not solvable. If $G$ has an abelian Sylow p-subgroup, then $G$ is a doubly transitive Frobenius group whose Frobenius kernel $R$ is an elementary abelian $r$-group of order $r^{2}$ where either $(p, r)=(7,29)$ or $(29,59)$, and a Frobenius complement $H \cong \mathrm{SL}_{2}(5) \times \mathbb{Z} / p$. In particular, $G$ satisfies conclusion (iv) of Theorem 1.1.

Proof. Let $P$ be a Sylow $p$-subgroup of $G$. By the Itô-Michler theorem, $P$ is not normal. Since $P$ is not normal in $G$, we have that $P$ does not centralize $F^{*}(G)$.

First assume that $R$ is a nilpotent normal $p^{\prime}$-subgroup of $G$ with $[P, R] \neq 1$. So $P$ does not commute with $R / \Phi(R)$. If $\gamma$ is a nontrivial linear character of $R$ vanishing on $\Phi(R)$ not fixed by $P$, then every constituent of $\gamma_{R}^{G}$ has dimension divisible by $p$, whence $G$ acts transitively on the nontrivial linear characters of $R / \Phi(R)$ not fixed by $P$. This implies that on a simple $G$-quotient $M$ of $R / \Phi(R)$, we have $G$ acting transitively on the nontrivial elements, whence [Liebeck 1987, Appendix 1] and the fact that $G$ is $p$-solvable implies that the action of $G$ on $M$ is $\mathrm{SL}_{2}(5) \times C$, where $C$ is a cyclic group of order divisible by $p,|M|=r^{2}$ with $(p, r)=(7,29)$ or $(29,59)$. Write $M=R / R_{0}$. If $P$ does not centralize $R_{0}$, then we can induce a nontrivial linear character of $R_{0}$ and obtain a different irreducible character of degree divisible by $p$ (it will have a different kernel), a contradiction. Thus, $P$ commutes with $R_{0}$. So $P$ acts as nontrivial scalars on $M$ and so $M$ does not commute with $\left[R, R_{0}\right]$ unless it is trivial. So $R_{0}=1$ and $|R|=r^{2}$.

Suppose $P$ does not commute with $F(G)$. We claim that this implies that $F^{*}(G)$ has order $r^{2}$ as above. If $E(G) \neq 1$, then clearly there is a character of $F^{*}(G)$ nontrivial on $E(G)$ that is not fixed by $P$ (if $P$ centralizes $E(G)$, then just take some character of $F^{*}(G)$ that is nontrivial on $\mathbf{O}_{r}(G)$ and $E(G)$; if $P$ does not 
centralize $E(G)$, just take a character of $E(G)$ not fixed by $P$ ) and inducing this up gives a contradiction.

So $F^{*}(G)$ of order $r^{2}$ implies that $F^{*}(G)$ is its own centralizer and we already saw that $G / F^{*}(G) \cong \mathrm{SL}_{2}(5) \times \mathbb{Z} / p$, whence the result.

So we may assume that $P$ commutes with $F(G)$. Then since $P$ is not normal in $G, P$ does not commute with $E(G)$. If $F^{*}(G) \neq E(G)$, then as above there will be at least two $G$-orbits of characters of $G$ of degree divisible by $p$ with distinct kernels. So $F^{*}(G)=E(G)$. We show that this cannot happen. There is no harm in passing to $G / \mathbf{Z}(E(G))$, and so we may assume that $E(G)$ is a direct product of nonabelian simple groups. The result now follows by Lemma 1.11.

Nonabelian Sylow p-subgroup. In this subsection, we complete the proof of Theorem 1.1 by proving it under the hypothesis that $G$ is $p$-solvable but not solvable and has a nonabelian Sylow $p$-subgroup. We will show that $G$ has to satisfy conclusion (vi) of Theorem 1.1. We also obtain further restrictions on the structure of $G$ in this situation.

Lemma 1.13. Let $p$ be a prime, and let $G$ be a group having exactly one irreducible character whose degree is divisible by $p$. Suppose that $G$ is $p$-solvable, but not solvable. Let $P$ be a Sylow p-subgroup of $G$. If $P$ is nonabelian, then $P$ is normal in $G$.

Proof. Suppose not. Suppose that $\mathbf{O}_{p}(G) \neq 1$ and let $Q$ be a minimal normal $p$-subgroup of $G$. If $P / Q$ is nonabelian, then by induction $P / Q$ is normal in $G / Q$, whence $P$ is normal.

So $P / Q$ is abelian, and recall that $P$ is not normal in $G$. By the Itô-Michler theorem, $G / Q$ has a character of degree divisible by $p$, and by hypothesis this character is unique. Since $G / Q$ is $p$-solvable and not solvable, we may apply Theorem 1.12, and $G / Q$ is as given there (in particular, $p=7$ or 29). Also, there is a normal subgroup $Q R$ where $R$ has order $r^{2}$ (with $r=29$ or 59 depending upon $p$ ).

If $R$ does not centralize $Q$, then as the element of order $p$ in $G / Q$ acts centrally on $R$ (that is, as scalars), we see that on $Q$, the dimension of the fixed space of a Sylow $p$-subgroup of $G$ on $Q$ is $(1 / p) \operatorname{dim} Q(Q$ is a free module for $P / Q)$ see Theorem 15.16 of [Isaacs 1976]. The number of Sylow $p$-subgroups in $G$ is $|R|=r^{2}$ (since $G=N_{G}(P) R$ ). Thus, the total number of points fixed by a Sylow $p$-subgroup of $G$ on $Q$ is less than $r^{2} p^{d / p}$, where $|Q|=p^{d}$.

Easily, we see that for both choices of $r$ and $p$ that $r^{2} p^{d / p}<p^{d}$, and so there exists an element of $Q$ (and similarly $Q^{*}$ ) that is fixed by no $p$-element outside $Q$. Thus, there is a linear character $\chi$ of $Q$ whose $G$-orbit has size a multiple of $p$, whence any irreducible constituent of $\chi^{G}$ has dimension a multiple of $p$ and so 
there are at least two irreducible characters of degree a multiple of $p$ (one with $Q$ in the kernel, one with $Q$ not in the kernel).

Suppose that $R$ centralizes $Q$. Then $P$ is central in $G / R Q$ and since $Q$ is an irreducible $G / R Q$-module, $P$ must act trivially on $Q$, whence $Q \leq \mathbf{Z}(P)$. Since $P / Q$ is cyclic, this implies that $P$ is abelian, a contradiction.

So we may assume that $\mathbf{O}_{p}(G)=1$. Let $N$ be a minimal normal subgroup. So $N$ is a $p^{\prime}$-group. Let $\chi$ be any character of $N$. If $P$ is not contained in the inertia group of $\chi$, then every irreducible constituent of $\chi_{N}^{G}$ has dimension divisible by $p$. Since some irreducible character of $G / N$ has dimension divisible by $p$, we have a contradiction. Indeed, the argument shows that $P$ centralizes $\mathbf{O}_{p^{\prime}}(G)$. Thus, $F^{*}(G)$ cannot be a $p^{\prime}$-group, whence $\mathbf{O}_{p}(G) \neq 1$. This completes the proof.

We now come to the proof of Theorem 1.1 under the additional hypothesis that $G$ is $p$-solvable but not solvable and that $G$ has a nonabelian Sylow $p$-subgroup. Combining Theorems 1.9, 1.12, and 1.14, we have a proof of Theorem 1.1.

Theorem 1.14. Let $p$ be a prime, and let $G$ be a group having exactly one irreducible character whose degree is divisible by $p$. Suppose that $G$ is p-solvable, but not solvable. If $G$ has a nonabelian Sylow p-subgroup, then $G$ satisfies conclusion (vi) of Theorem 1.1. Furthermore, $p=11,29$, or $59 ;\left|P^{\prime}\right|=p^{2}$; and $G / P \cong \mathrm{SL}_{2}(5) \times \mathbb{Z} / c(p)$, where $c(11)=1, c(29)=7$, and $c(59)=29$.

Proof. By Lemma 1.13, $P$ is normal. Applying Lemma 1.8, $G$ satisfies conclusion (vi) of Theorem 1.1. Let $P$ be the Sylow $p$-subgroup, and let $H$ be a $p$-complement in $G$. We know that $P^{\prime} H$ is a doubly transitive Frobenius group. It follows that $H$ is a Frobenius complement. Since $H$ is nonsolvable, it follows by [Passman 1968, 20.2] that $|\mathbf{Z}(P)|=p^{2}$ with $p=11,29$, or 59 and $H=\mathrm{SL}_{2}(5) \times C$, where $C$ is cyclic of order $c(p)$.

Examples with exceptional doubly transitive Frobenius groups. In this subsection, we consider groups $G$ that have the form of a coprime semidirect product of a group $H$ acting on a semi-extraspecial $p$-group $P$ where $|\mathbf{Z}(P)|=p^{2}$ and $H$ is acting faithfully and irreducibly on $\mathbf{Z}(P)$. We find the possible values for $\left|P: P^{\prime}\right|$ when $H$ is either $\mathrm{SL}_{2}(3)$ or $\mathrm{SL}_{2}(5)$, and we find examples showing that each of the possible values occur. This will yield examples where $G$ is a group that satisfies conclusion (vi) of Theorem 1.1 where $H P^{\prime}$ is any of the exceptional two-transitive Frobenius groups. In particular, this gives examples of groups $G$ where $G$ is a $p$-solvable group that is not solvable with a nonabelian Sylow subgroup $P$ and exactly one irreducible character whose degree is divisible by $p$.

The key to this solution is trying to find $\mathbb{F}_{p} H$-modules $V$ so that $\wedge^{2}(V)$ contains a 2-dimensional $H$-submodule $W$ such that $W^{\#}$ consists of nondegenerate alternating forms on $V$. There is also is an intriguing connection with the McKay 
correspondence and the Dynkin diagram of affine extended $E_{8}$ when $H=\mathrm{SL}_{2}(5)$ and of affine extended $E_{6}$ when $H=\mathrm{SL}_{2}$ (3). (For more details on the McKay correspondence and Dynkin diagrams, see [McKay 1980] or [Steinberg 1985].) This was used in our initial approach to the problem but it is not needed in the solution.

In this section we address a slightly more general problem than is addressed in the remainder of the paper. We assume we have a group $H$ with $\mathbf{Z}(H) \leq H^{\prime}$ and $|Z(H)|=2$ that is acting faithfully on a $p$-group $P$ that satisfies the following conditions. In the examples needed for this paper, we will have $H=\mathrm{SL}_{2}(3)$ or $H=\mathrm{SL}_{2}(5)$.

Our hypotheses are as follows:

(i) $p$ is a prime that is coprime to $|H|$.

(ii) $P$ is a semi-extraspecial $p$-group with $\left|P: P^{\prime}\right|=p^{a}$ and $Z=\mathbf{Z}(P)=P^{\prime}$ has order $p^{2}$.

(iii) $H$ acts on $P$ with $Z$ a faithful irreducible $H$-module (and we fix the isomorphism type).

Notice that $H$ acting faithfully on $Z$ implies that $H$ is isomorphic to a subgroup of $\mathrm{SL}_{2}(p)$. For $H=\mathrm{SL}_{2}$ (3), this does not imply any further restrictions on $p$, but when $H=\mathrm{SL}_{2}(5)$, this implies that $p \equiv \pm 1 \bmod 5$. Set $V=P / Z$, and we view $V$ as a module for $H$. We will determine the set of all positive integers $a$ so that $|V|=p^{a}$ when $H=\mathrm{SL}_{2}(3)$ and $H=\mathrm{SL}_{2}(5)$. The particular values that occur depend on the residue class of $p$ modulo 12 when $H=\mathrm{SL}_{2}(3)$ and modulo 60 when $H=\mathrm{SL}_{2}(5)$. In the next section, we will construct enough examples to show that there exists an example for each of the possible dimensions. The same techniques would essentially allow us to classify all the possible groups, but we do not pursue this here. Let $V_{1}$ and $V_{-1}$ denote the eigenspaces of the action of $\mathbf{Z}(H)$ on $V$. Let $P_{1}$ and $P_{-1}$ denote the inverse images of $V_{1}$ and $V_{-1}$ in $P$.

Lemma 1.15. Assume $P$ and $H$ satisfy the given hypotheses and $P_{1}$ and $P_{-1}$ are defined as above. Then $P_{1}$ and $P_{-1}$ are abelian, $a=2 b$ is even, and $\left|P_{1}\right|=\left|P_{-1}\right|=p^{b+2}$. Proof. Note that $Z(H)$ acts trivially on $\left[P_{\epsilon}, P_{\epsilon}\right]$ for $\epsilon=1$ or $\epsilon=-1$. Since $Z(H)$ does not active trivially on $Z$, we must have $\left[P_{\epsilon}, P_{\epsilon}\right] \cap Z=1$. Thus, $P_{1}$ and $P_{-1}$ are abelian. Since $P / Z_{1}$ is extraspecial, this implies that $\left|P_{ \pm 1}: Z\right| \leq p^{a / 2}$. Since $p^{a}=\left|V_{1}\right|\left|V_{-1}\right|$, we have that $a=2 b$ for some integer $b$ and $\left|P_{ \pm 1}: Z\right|=p^{b}$. The conclusion now follows.

Given a subgroup $1<Z_{1}<Z$, we obtain an element of $\left(\bigwedge^{2} V\right)^{*}=\operatorname{Hom}\left(\bigwedge^{2} V, \mathbb{F}_{p}\right)$ (namely the homomorphism $a Z \wedge b Z \mapsto[a, b] Z_{1}$ ). The condition that $P / Z_{1}$ is extraspecial is equivalent to saying that this element is nondegenerate. The subspace of $\left(\wedge^{2} V\right)^{*}$ generated by these elements yields a 2-dimensional irreducible 
$H$-invariant subspace of $\left(\bigwedge^{2} V\right)^{*}$ such that each nonzero element is nondegenerate. In the next section, we will use the fact that if such a 2-dimensional space exists, then we can construct $P$ satisfying the above conditions.

The existence of a group $P$ such that $H$ acts as above implies that an $H$-invariant subspace $W$ of $\left(\wedge^{2} V\right)^{*}$ exists such that $W$ is two dimensional and every nonzero element of $W$ is nondegenerate. We choose a basis for $V$ consisting of bases for $V_{1}$ and $V_{-1}$. Representing the elements in $W$ as matrices in terms of this basis for $V$, we obtain matrices of the form

$$
f(D):=\left(\begin{array}{cc}
0 & D \\
-D^{T} & 0
\end{array}\right),
$$

where $D$ is some element of $M_{n}\left(\mathbb{F}_{p}\right)$. The fact that the nonzero elements of $W$ are nondegenerate is equivalent to saying that $D$ is nonsingular for all the matrices $D$ such that $f(D) \in W$.

We can conjugate and find a basis for $W$ of the form $\{f(I), f(A)\}$ for some matrix $A$. Let $\tilde{W}$ be the $\mathbb{F}_{p}$-span of $\{I, A\}$. Observe that $f$ is an isomorphism of the vector spaces $W$ and $\tilde{W}$. Moreover, the condition that all of the nonzero matrices in $\tilde{W}$ are nonsingular is precisely equivalent to the condition that $A$ has no eigenvalues in $\mathbb{F}_{p}$.

Let $\tau$ denote the inverse transpose map. We may write the action of $H$ on $W$ as $\operatorname{diag}\left(\rho_{1}(h), \rho_{2}(h)^{\tau}\right)$, where $\rho_{1}$ is the representation of $H$ afforded by $V_{1}$ and $\rho_{2}$ is the representation of $H$ afforded by $V_{2}$. It follows that $\rho_{1}$ has a kernel containing $\mathbf{Z}(H)$ and that $\rho_{2}$ is a faithful representation of $H$. The fact that $W$ is $H$-invariant is precisely equivalent to saying that

$$
\rho_{1}(h) W \rho_{2}(h)^{-1}=W
$$

for every element $h \in H$. Since we are taking $\{f(I), f(A)\}$ as the basis for $W$, we see that $\rho_{1}(h) \rho_{2}(h)^{-1}=\rho_{1}(h) I \rho_{2}(h)^{-1} \in \tilde{W}$ and $\rho_{1}(h) A \rho_{2}(h)^{-1} \in \tilde{W}$ for all $h \in H$. Because $\rho_{1}(h) I \rho_{2}(h)^{-1} \in \tilde{W}$, there is a map $\phi$ from $H$ to $\tilde{W}$ define by $\phi(h)=\rho_{1}(h) I \rho_{2}(h)^{-1}$. This satisfies $\rho_{1}(h) I=\phi(h) \rho_{2}(h)$ for all $h \in H$, and substituting, we obtain $\rho_{1}(h) A \rho_{2}(h)^{-1}=\phi(h) \rho_{2}(h) A \rho_{2}(h)^{-1}$. Since $\phi(h) \in \tilde{W}$, this implies that $\rho_{2}(h) A \rho_{2}(h)^{-1} \in \tilde{W}$. It follows that conjugation by $\rho_{2}(h)$ preserves the algebra $R=\mathbb{F}_{p}[A]$, and this conjugation action is therefore an algebra automorphism of $R$. We define $\rho: H \rightarrow \tilde{W}$ by $\rho(h)=\rho_{1}(h) A \rho_{2}(h)^{-1}$, and note that $\rho(h)=\phi(h) \rho_{2}(h) A \rho_{2}(h)^{-1}$. Since the nonzero elements of $\tilde{W}$ are nonsingular (i.e., invertible), it follows that $\phi(h) \in R^{*}$ for all $h \in H$, and this implies that the image of $H$ under $\rho$ is contained in $R^{*} \operatorname{Aut}(R)$, where $R^{*}$ is the set of units in $R$.

Note that $\rho(H)$ acts by permutations on the primitive idempotents of $R$. Thus, we can decompose $R=R_{1} \times \cdots \times R_{m}$, where each $R_{i}$ is a subspace of $R$ generated by an orbit of $\rho(H)$ on the primitive idempotents of $R$. We have $\operatorname{dim} R=\sum_{i=1}^{m} \operatorname{dim} R_{i}$. 
We now work with $R_{1}$. We know that $\rho(H)$ acts transitively on the primitive idempotents of $R_{1}$. We have $R_{1}=K\left[t_{1}\right] / t_{1}^{e} \times \cdots \times K\left[t_{s}\right] / t_{s}^{e}$, where $K$ is a nontrivial extension field of $\mathbb{F}_{p}$. The fact that $K$ is a nontrivial extension comes from the fact that the eigenvalues of $A$ do not lie in $\mathbb{F}_{p}$. Since $Z(H)$ acts like -1 , it stabilizes the subspaces generated by each of the $t_{i}$ 's.

Let $H_{1}$ denote the stabilizer of the subspace generated by $t_{1}$ which we call $L_{1}$, and note that $s=\left|H: H_{1}\right|$. Then $\rho_{2}\left(H_{1}\right)$ acts on $L_{1}:=K\left[t_{1}\right] / t_{1}^{e}$, and again, the element of order 2 in $Z(H) \leq H_{1}$ acts as -1 . Thus, $H_{1}$ acts faithfully on $L_{1}$. Moreover, the action of $H_{1}$ on $L_{1}$ embeds in $L_{1}^{*} \operatorname{Aut}\left(L_{1}\right)$. Because $H_{1}$ has order prime to $p$, we see that in fact $H_{1}$ embeds in $K^{*} \operatorname{Aut}(K)$, and this implies that $H_{1}$ is either cyclic or metacyclic. If $\left|H_{1}\right|=2$, then since $K$ is a nontrivial extension of $\mathbb{F}_{p}$, we see that $H_{1}$ does not act irreducibly on $L$, and so we conclude that $\left|H_{1}\right| \neq 2$.

Suppose $H_{1}$ is cyclic of order $2 q$, where $q$ is an odd prime. If $q$ divides $p-1$, then $H_{1}$ will not act irreducibly on $L_{1}$, so $q$ does not divide $p-1$. Recall that $H_{1}$ is isomorphic to a subgroup of $\mathrm{SL}_{2}(p)$. Thus, it must be that $q$ divides $p+1$. This implies that $\left[K: \mathbb{F}_{p}\right]$ is even and $p \equiv-1 \bmod q$.

Now, suppose $H_{1}$ is cyclic of order 4 . If 4 does not divide $p-1$, then it is not difficult to see that $\left[K: \mathbb{F}_{p}\right]$ must be even. Suppose 4 does divide $p-1$. For $H_{1}$ to be acting irreducibly on $L_{1}$, it must be that a generator of $H_{1}$ is the product of a nontrivial element of $L_{1}^{*}$ with a nontrivial element of $\operatorname{Aut}\left(L_{1}\right)$. In particular, this implies that 2 divides $\left[K: \mathbb{F}_{p}\right]$.

If $H_{1}$ is nonabelian, then since $H_{1}$ is metacyclic and contained in $\operatorname{SL}_{2}(p)$, we conclude that $H_{1}^{\prime}$ is cyclic and has index 2 . This implies that 2 divides $\left[K: \mathbb{F}_{p}\right.$ ]. If $H_{1}$ contains a subgroup isomorphic to the quaternions, then $p \equiv-1 \bmod 4$ since $K^{*} \operatorname{Aut}(K)$ does not contain any subgroups isomorphic to the quaternions when $p \equiv 1 \bmod 4$. We now assume that $H_{1}$ does not contain any subgroups isomorphic to the quaternions. Since $H_{1}$ is a subgroup of $\mathrm{SL}_{2}(p)$, this implies that the Sylow 2-subgroups of $H_{1}$ are abelian, and so $\left|H_{1}\right|$ is divisible by some odd prime $q$. Since $H_{1}$ is acting irreducibly, we see that $H_{1} / \mathbf{Z}(H)$ is contained in a dihedral group of order $2(p+1)$. This implies $H_{1}^{\prime}$ contains no elements of odd order whose order divides $p-1$. In particular, we must have $q$ divides $p+1$ so $p \equiv-1 \bmod q$.

Theorem 1.16. Let $P$ be an semi-extraspecial p-group with $|\mathbf{Z}(P)|=p^{2}$ and $p>5$ is a prime. Let a be the even integer so that $|P: \mathbf{Z}(P)|=p^{a}$. Suppose $H=\mathrm{SL}_{2}(5)$ acts via automorphisms on $P$ such that $H$ is acting faithfully and irreducibly on $Z(H)$. Then $p \equiv \pm 1 \bmod 5$ and the following holds:

(i) If $p \equiv 1 \bmod 60$, then $a=120 x$, where $x$ is a positive integer.

(ii) If $p \equiv 11 \bmod 60$, then $a=40 x+60 y$, where $x$ and $y$ are nonnegative integers whose sum is positive. 
416 D. Goldstein, R. Guralnick, M. Lewis, A. Moretó, G. Navarro and P. H. Tiep

(iii) If $p \equiv 19 \bmod 60$, then $a=24 x+60 y$, where $x$ and $y$ are nonnegative integers whose sum is positive.

(iv) If $p \equiv 29 \bmod 60$, then $a=24 x+40 y$, where $x$ and $y$ are nonnegative integers whose sum is positive.

(v) If $p \equiv 31 \bmod 60$, then $a=60 x$, where $x$ is a positive integer.

(vi) If $p \equiv 41 \bmod 60$, then $a=40 x$, where $x$ is a positive integer.

(vii) If $p \equiv 49 \bmod 60$, then $a=24 x$, where $x$ is a positive integer.

(viii) If $p \equiv 59 \bmod 60$, then $a=24 x+40 y+60 z$, where $x, y$, and $z$ are nonnegative integers whose sum is positive.

Proof. We consider the possibility for a subgroup $H_{1}$ of $\mathrm{SL}_{2}(5)$ within the context of the previous argument. Since $H_{1}$ is cyclic or metacyclic and $\mathbf{Z}(H) \leq H_{1}$, we see that $s$ is the index of a proper subgroup of $\mathrm{A}_{5}$ and $s \neq 5$, whence $s=6,10,12,15,20,30$, or 60 . Since $\left|H_{1}\right| \neq 2$, we conclude that $s \neq 60$. We see that one of the following holds:

(i) $s=6, H_{1}=5.4,\left[K: \mathbb{F}_{p}\right]$ is even, $p \equiv-1 \bmod 5$, and $\operatorname{dim} R_{1}$ is a multiple of 12.

(ii) $s=10, H_{1}=3.4,\left[K: \mathbb{F}_{p}\right]$ is even, $p \equiv-1 \bmod 3$, and $\operatorname{dim} R_{1}$ is a multiple of 20 .

(iii) $s=12, H_{1}$ is cyclic of order $10,\left[K: \mathbb{F}_{p}\right]$ is even, $p \equiv-1 \bmod 5$, and $\operatorname{dim} R_{1}$ is a multiple of 24 .

(iv) $s=15, H_{1}=Q_{8},\left[K: \mathbb{F}_{p}\right]$ is even, $p \equiv-1 \bmod 4$, and $\operatorname{dim} R_{1}$ is a multiple of 30 .

(v) $s=20, H_{1}$ is cyclic of order $6,\left[K: \mathbb{F}_{p}\right]$ is even, $p \equiv-1 \bmod 3$ and $\operatorname{dim} R_{1}$ is a multiple of 40 .

(vi) $s=30, H_{1}$ is cyclic of $\operatorname{order} 4,\left[K: \mathbb{F}_{p}\right]$ is even, and $\operatorname{dim} R_{1}$ is a multiple of 60.

Since $\operatorname{dim} V_{1}=\sum \operatorname{dim} R_{i}$, this gives possible dimensions for $V_{1}$ depending upon the congruences of $p$ modulo 30. Note that $a=\operatorname{dim} V=2 \operatorname{dim} V_{1}$. This gives the stated conclusion.

We saw in Theorem 1.14 the primes $p$ that arise in our case are $p=11,29$, or 59 , and for these primes we obtain the following possibilities.

Corollary 1.17. Assume the hypotheses of Theorem 1.16.

(i) If $p=11$, then $a=40 x+60 y$ for some nonnegative integers $x$ and $y$.

(ii) If $p=29$, then $a=24 x+40 y$ for some nonnegative integers $x$ and $y$.

(iii) If $p=59$, then $a=24 x+40 y+60 z$ for some nonnegative integers $x, y$, and $z$. 
In the solvable examples, we have the group $\mathrm{SL}_{2}(3)$ acting on $P$. Thus, we have the following.

Theorem 1.18. Let $P$ be an semi-extraspecial $p$-group with $|\mathbf{Z}(P)|=p^{2}$ and $p>3$ is a prime. Let a be the even integer so that $|P: \mathbf{Z}(P)|=p^{a}$. Suppose $H=\mathrm{SL}_{2}(3)$ acts via automorphisms on $P$ such that $H$ is acting faithfully and irreducibly on $Z(H)$. Then the following holds:

(i) If $p \equiv 1 \bmod 12$, then $a=24 x$, where $x$ is a positive integer.

(ii) If $p \equiv 5 \bmod 12$, then $a=16 x+24 y$, where $x$ and $y$ are nonnegative integers whose sum is positive.

(iii) If $p \equiv 7 \bmod 12$, then $a=12 x$, where $x$ is a positive integer.

(iv) If $p \equiv 11 \bmod 12$, then $a=12 x+16 y$, where $x$ and $y$ are nonnegative integers whose sum is positive.

Proof. We consider the possibility for a subgroup $H_{1}$ of $\mathrm{SL}_{2}(3)$ within the context of the previous argument. Since $H_{1}$ is cyclic or metacyclic and $\mathbf{Z}(H) \leq H_{1}$, we see that $s$ is the index of a proper subgroup of $\mathrm{A}_{4}$, whence $s=3,4,6$, or 12 . Since $\left|H_{1}\right| \neq 2$, we conclude that $s \neq 12$. We see that one of the following holds:

(i) $s=3, H_{1}=Q_{8},\left[K: \mathbb{F}_{p}\right]$ is even, $p \equiv-1 \bmod 4$, and $\operatorname{dim} R_{1}$ is a multiple of 6 .

(ii) $s=4, H_{1}$ is cyclic of order $6,\left[K: \mathbb{F}_{p}\right]$ is even, $p \equiv-1 \bmod 3$, and $\operatorname{dim} R_{1}$ is a multiple of 8 .

(iii) $s=6, H_{1}$ is cyclic of order $4,\left[K: \mathbb{F}_{p}\right]$ is even, and $\operatorname{dim} R_{1}$ is a multiple of 12 . Since $\operatorname{dim} V_{1}=\sum \operatorname{dim} R_{i}$, this gives possible dimensions for $V_{1}$ depending upon the congruences of $p$ modulo 30 . Note that $a=\operatorname{dim} V=2 \operatorname{dim} V_{1}$. This gives the stated conclusion.

We will also construct examples for the solvable exceptional 2-transitive Frobenius groups. (For a list of the exceptional 2-transitive Frobenius groups, see Remark XII.9.5 in [Huppert and Blackburn 1982]. We note that matrix generators for these groups seem to be correct, even though the descriptions in (b) and (d) are not. In particular, in (b) $H \cong \widehat{\mathrm{GL}_{2}(3)}$, which is isoclinic to but not isomorphic to $\mathrm{GL}_{2}$ (3), and in $(\mathrm{d}) H \cong \widetilde{\mathrm{GL}_{2}(3)} \times \mathbb{Z} / 11$. The matrix groups are also listed in [Huppert 1957].) The primes $p$ that arise are $p=5,7,11$, and 23. For these primes, we obtain the following.

Corollary 1.19. Assume the hypotheses of Theorem 1.18.

(i) If $p=5$, then $a=16 x+24 y$, where $x$ and $y$ are nonnegative integers whose sum is positive.

(ii) If $p=7$, then $a=12 x$, where $x$ is a positive integer. 


\begin{tabular}{rccc}
\hline$p$ & $H$ & $a$ & $a$ \\
\hline 5 & $\mathrm{SL}_{2}(3)$ & $16 x+24 y$ & $16 w, w \geq 2$ \\
7 & $\widehat{\mathrm{GL}_{2}(3)}$ & $12 x$ & $12 w, w \geq 1$ \\
11 & $\mathrm{SL}_{2}(3) \times \mathbb{Z} / 5$ & $12 x+16 y$ & $4 w, w \neq 1,2,5$ \\
11 & $\mathrm{SL}_{2}(5)$ & $40 x+60 y$ & $20 w, w \geq 2$ \\
23 & $\mathrm{GL}_{2}(3) \times \mathbb{Z} / 11$ & $12 x+16 y$ & $4 w, w \neq 1,2,5$ \\
29 & $\mathrm{SL}_{2}(5) \times \mathbb{Z} / 7$ & $24 x+40 y$ & $8 w, w \geq 3, w \neq 4,7$ \\
59 & $\mathrm{SL}_{2}(5) \times \mathbb{Z} / 29$ & $24 x+40 y+60 z$ & $4 w, w \geq 6$, \\
\hline
\end{tabular}

Table 1. Values in Theorem 1.20.

(iii) If $p=11$, then $a=12 x+16 y$, where $x$ and $y$ are nonnegative integers whose sum is positive.

(iv) If $p=23$, then $a=12 x+16 y$, where $x$ and $y$ are nonnegative integers whose sum is positive.

We will now construct examples showing that there are groups satisfying our hypotheses.

Theorem 1.20. For each prime $p$, group $H$, and value $a$ in Table 1 , there exist groups $P$ and $G$ so that $P$ is a semi-extraspecial p-group with $\left|P^{\prime}\right|=p^{2}$ and $\left|P: P^{\prime}\right|=p^{a}$ such that $H$ acts faithfully by automorphisms on $P$ and $G$ is the resulting semidirect product. In addition, $\operatorname{Irr}(G)$ has a unique character with degree divisible by $p$ and $P^{\prime} H$ is a two-transitive Frobenius group.

Proof. Suppose first that $P$ and $Q$ are semi-extraspecial $p$-groups with $|\mathbf{Z}(P)|=$ $|\mathbf{Z}(Q)|=p^{2}$, and $\operatorname{dim} P / \mathbf{Z}(P)=a_{1}$ and $\operatorname{dim} Q / \mathbf{Z}(Q)=a_{2}$. Suppose that the group $H$ acts via automorphisms on $P$ and $Q$ so that the action of $H$ on $\mathbf{Z}(P)$ is isomorphic to its action on $\mathbf{Z}(Q)$ and this action is faithful and irreducible. Let $R$ be the central product of $P Q$ (that is, identifying $\mathbf{Z}(Q)$ and $\mathbf{Z}(P))$. Then $|\mathbf{Z}(R)|=p^{2}$ and $R / Z_{1}$ is extraspecial for any proper nontrivial subgroup $Z_{1}$ of $\mathbf{Z}(R)$ since $R / Z_{1}$ is the central product of the two extraspecial groups $P / Z_{1}$ and $Q / Z_{1}$. It follows that $R$ is semiextraspecial and $\operatorname{dim} R / \mathbf{Z}(R)=a_{1}+a_{2}$. Note that $H$ will act via automorphisms on $R$ and that the action of $H$ on $\mathbf{Z}(R)$ is faithful and irreducible. This shows that the set of dimensions that can occur is closed under sums. In particular, the entries in the first column for values for $a$ are linear combinations, and it suffices to show that there exist examples for the generators of these linear combinations.

Let $p$ and $H$ be entries for the first two columns of some row in Table 1, and let $a$ be one of the generators of the third column. Observe that $H$ is isomorphic to a subgroup of $\mathrm{GL}_{2}(p)$, so it has a faithful 2-dimensional module $Z$ over $\mathbb{F}_{p}$. Let $\mathrm{H}_{2}$ be a subgroup of $H$ containing $Z(H)$ whose index is $a / 4$. We have $H_{2}=H_{1} \times C$. 
We take $C=Z / c$, where $c=1$ when $p$ is 5 or 7 , or $p=11$ and $H$ is not solvable. Otherwise, $c=5$ when $p=11$ and $H$ is solvable, $c=11$ when $p=23, c=7$ when $p=29$, and $c=29$ when $p=59$. When $H$ is not solvable, then $H_{1}$ will have order $4 r$, where $r=2,3$, or 5 , and we note that $p$ satisfies $p \equiv-1 \bmod 2 r$. When $H$ is solvable, we have $\left|H_{1}\right|=4$ or 6 when $p=5,\left|H_{1}\right|=16$ when $p=7,\left|H_{1}\right|=6$ or 8 when $p=11$, and $\left|H_{1}\right|=12$ or 16 when $p=23$.

Let $X$ be the 2-dimensional $\mathbb{F}_{p} H_{2}$-module where the image of $H_{1}$ is generated by an involution of determinant -1 and the module for $C$ is the restriction of $Z$. Let $Y$ be the 2-dimensional $\mathbb{F}_{p} H_{2}$-module which is the restriction of $Z$ to $H_{2}$. We can view $H_{1}$ as being contained in a subgroup of order $2(p+1)$ in $\mathrm{GL}_{2}(p)$. Notice also that $C$ will be isomorphic to a subgroup of the center of $\mathrm{GL}_{2}(p)$, and thus $H_{2}=H_{1} \times C$ can be thought of as normalizing a given nonsplit torus in $\mathrm{GL}_{2}(p)$. It follows that $\operatorname{Hom}(X, Y)$ contains a 2-dimensional invariant $H_{2}$-submodule $U \cong Y$ with $U$ consisting of invertible elements (namely the algebra generated by the nonsplit torus which is a quadratic extension of $\mathbb{F}_{p}$ ).

It is straightforward to compute (by Frobenius reciprocity) that $U^{H}$ contains a unique submodule $W$ isomorphic to $Z$. Note that $U^{H} \leq \operatorname{Hom}(X, Y)_{H_{2}}^{H} \leq$ $\operatorname{Hom}\left(X^{H}, Y^{H}\right)$. Since $U$ consists of invertible elements, it is straightforward to see that $W$ does as well. We take $V=X_{H_{2}}^{H}+Y_{H_{2}}^{H}$. We identify $\operatorname{Hom}\left(X_{H_{2}}^{H}, Y_{H_{2}}^{H}\right)$ with a submodule of $\left(\wedge^{2} V\right)^{*}$ as follows. Let $\left\{e_{1}, \ldots, e_{n}\right\}$ be a basis for $X^{H}$ and $\left\{f_{1}, \ldots, f_{n}\right\}$ be a basis for $y^{H}$. Given an element $\phi \in \operatorname{Hom}\left(X^{H}, y^{H}\right)$, we define the matrix $D=D_{\phi}$ whose $(i, j)$-entry is $\phi\left(e_{i}, f_{j}\right)$. This defines an element

$$
\left(\begin{array}{cc}
0 & D \\
-D^{T} & 0
\end{array}\right) \in\left(\wedge^{2} V\right)^{*}
$$

This yields $W$ embedded as a submodule of $\left(\bigwedge^{2} V\right)^{*}$, and as we mentioned earlier, this allows us to construct a semi-extraspecial $p$-group $P$ so that $P / \mathbf{Z}(P) \cong V$ and $\mathbf{Z}(P) \cong W \cong Z$ (as $H$-modules) with the desired properties.

This shows that all possible dimensions given in the previous section do occur and completes the proof of the main theorem.

\section{Non- $p$-solvable groups}

The goal of this section is to prove the following theorem. Notice that this includes the non- $p$-solvable portion of the main theorem. We do get somewhat more information. In particular, we determine precisely the degree of the one character whose degree is divisible by $p$.

Theorem 2.1. Let $p$ be a prime and let $G$ be a finite non-p-solvable group. Then $G$ has exactly one complex irreducible character of degree (say D) divisible by $p$ if and only if one of the following holds. 
(i) $D=q=p^{a} \geq 4$, a power of $p$. Furthermore, $G=\operatorname{PSL}_{2}(q), \mathrm{SL}_{2}(q)$, or $G / V=\mathrm{SL}_{2}(q)$, where $V$ is a minimal normal elementary abelian $p$-subgroup of $G$ having order $q^{2}$ and can be viewed as a 2-dimensional irreducible module of $G / V$ over $\operatorname{End}_{G / V}(V) \cong \mathbb{F}_{q^{2}}$.

(ii) $(G, p, D)=\left(\mathrm{S}_{5}, 3,6\right),\left(M_{11}, 3,45\right)$.

For brevity, we will call $G$ an NP1-group (for a fixed prime $p$ ) if $G$ is a finite non- $p$-solvable group with a unique irreducible character $\chi$ of degree divisible by $p$, in which case we let $D:=\chi(1)$.

We make use of results regarding character degree graphs. The graph $\Delta(G)$ is the graph with vertex set $\rho(G)$, which is the set of primes dividing degrees in $\{\chi(1) \mid \chi \in \operatorname{Irr}(G)\}$. There is an edge between distinct primes $p$ and $q$ if $p q$ divides $\chi(1)$ for some $\chi \in \operatorname{Irr}(G)$. We will make use of the results regarding nonsolvable groups $G$ where $\Delta(G)$ is disconnected in [Lewis and White 2003] and [Manz et al. 1988]. We prove the following statement combining the results on disconnected graphs with some results of [Isaacs et al. 2009]:

Proposition 2.2. Let $G$ be an NP1-group. Then one of the following holds.

(i) $D=q=p^{a} \geq 4$ is a power of $p$, and $G / K=\operatorname{PSL}_{2}(q)$. Furthermore, $G=\operatorname{PSL}_{2}(q), \mathrm{SL}_{2}(q)$, or $G / V=\mathrm{SL}_{2}(q)$, where $V$ is a minimal normal elementary abelian p-subgroup $G$ of order $q^{2}$ and can be viewed as a 2dimensional irreducible module of $G / V$ over $\operatorname{End}_{G / V}(V) \cong \mathbb{F}_{q^{2}}$.

(ii) Let $U=\mathbf{O}_{p}(G)$ and $K / U=\mathbf{O}_{p^{\prime}}(G / U)$. Then $K$ is the p-solvable radical of $G$ and $U$ is abelian. If $N \leq K$ is a normal subgroup of $G$ and $1_{N} \neq \lambda \in \operatorname{Irr}(N)$, then the inertia group $I_{G}(\lambda)$ of $\lambda$ has index coprime to $p$ in $G$. Finally, $(G / K, p, D)$ is either $\left(\mathrm{S}_{5}, 3,6\right)$ or $\left(M_{11}, 3,45\right)$.

Proof. By Theorems A and C of [Isaacs et al. 2009], we know that if $U=\mathbf{O}_{p}(G)$ and $K / U=\mathbf{O}_{p^{\prime}}(G / U)$, then $K$ is the $p$-solvable radical of $G$ and $U$ is abelian. We also obtain from there the fact that if $N \leq K$ is a normal subgroup of $G$ and $1_{N} \neq \lambda \in \operatorname{Irr}(N)$, then the inertia group $I_{G}(\lambda)$ of $\lambda$ has index coprime to $p$ in $G$. Finally, that result also asserts that $\operatorname{soc}(G / K)=S / K=: \bar{S}$ is a nonabelian simple group of order divisible by $p$, and $p$ is coprime to $|G / S|$. By the Itô-Michler theorem, we may assume that the unique irreducible character $\chi$ of degree divisible by $p$ of $G$ is actually a character of $G / K$. In particular, every irreducible character of $K$ has degree coprime to $p$, whence the (unique) Sylow $p$-subgroup $U$ of $K$ must be abelian again by the Itô-Michler theorem. Next, let $N \leq K$ be normal in $G$ and $1_{N} \neq \lambda \in \operatorname{Irr}(N)$. Consider any $\rho \in \operatorname{Irr}(G \mid \lambda)$. Since $\rho_{N}$ contains $\lambda \neq 1_{N}$, $\operatorname{Ker}(\rho)$ cannot contain $N$, and so does not contain $K$. Thus $\rho \neq \chi$ and so has degree coprime to $p$. It follows that $\left[G: I_{G}(\lambda)\right]$ is coprime to $p$. 
Next, by Corollary 7.5 of [Isaacs et al. 2009] applied to $G / K$, one of the following possibilities occurs:

(a) $\bar{S} \cong \mathrm{PSL}_{2}(q)$ with $p \mid q$, and $D=q$.

(b) $\bar{S} \cong \mathrm{PSL}_{2}(q), q=r^{f}$ for some prime $r \neq p, 2<p \mid(q-\epsilon)$ for some $\epsilon= \pm 1$, and $D \mid(q-\epsilon) f$.

(c) $(G / K, p, D) \in\left\{\left(M_{11}, 3,45\right),\left(J_{1}, 3,120\right),\left(J_{1}, 5,120\right)\right\}$.

Note that $J_{1}$ has 3 irreducible characters of degree 120 . Hence, in the case of (c) we arrive at conclusion (ii).

Suppose now that we are in the case of (a). Consider the Steinberg character St of $S / K=\mathrm{PSL}_{2}(q)$, of degree $q$. By the main result of [Feit 1993], St extends to $G / K$. Hence $D=q$, and since $p$ divides the degree of no other character in $\operatorname{Irr}(G)$, this implies that $p$ is an isolated vertex in $\Delta(G)$, and so $\Delta(G)$ is disconnected. In [Manz et al. 1988], it is shown that $\Delta(G)$ has at most three connected components. In Theorems 4.1 and 6.4 of [Lewis and White 2003], it is shown that if $G$ is nonsolvable and $\Delta(G)$ has two or three connected components, then $G$ has a normal subgroup $N$ so that $G / N$ is abelian and $N$ is either $\operatorname{PSL}_{2}(q), \operatorname{SL}_{2}(q)$, or there is a normal subgroup $V$ so that $N / V \cong \operatorname{SL}_{2}(q)$ and $V$ is elementary abelian of order $q^{2}$ and $N / V$ acts transitively on the nonidentity elements of $V$. As before, the Steinberg character of $N$ will extend to $G$, and, by Gallagher's theorem, $G$ has at least $|G / N|$ irreducible characters of degree divisible by $q$. Since $G$ is an NP1group, it follows that $|G / N|=1$, whence $G=N$ and we arrive at conclusion (i).

From now on, we may assume that (b) holds. Since $p$ is coprime to $|G / S|$, all irreducible constituents $\alpha_{i}$ of $\chi_{S}$ have degree divisible by $p$. On the other hand, if $\beta \in \operatorname{Irr}(\bar{S})$ has degree divisible by $p$, then any $\gamma \in \operatorname{Irr}(G / K \mid \beta)$ also has degree divisible by $p$, and so $\gamma=\chi$ as $G$ is an NP1-group. Thus $\beta$ must be one of the $\alpha_{i}$. We have shown that $G / S$, and so $\operatorname{Aut}(\bar{S})$ as well, acts transitively on the set of irreducible characters of $\bar{S}$ of degree divisible by $p$. Also recall that $2<p \mid(q-\epsilon)$. Now we distinguish the following subcases.

(b1) $q \equiv-\epsilon(\bmod 4)$. In this case, $p$ divides $(q-\epsilon) / 2$, which is odd, and $\bar{S}$ has two irreducible (Weil) characters of degree $(q-\epsilon) / 2$. Now if $q \geq 7$, then $\bar{S}$ also has irreducible characters of degree $(q-\epsilon)$ which are obviously not Aut $(\bar{S})$-conjugate to the ones of degree $(q-\epsilon) / 2$. Hence $q=5, \epsilon=-1, p=3, \bar{S} \cong \mathrm{A}_{5}$, and $\mathrm{A}_{5} \leq G / K \leq \mathrm{S}_{5}$. Since $\mathrm{A}_{5}$ has two irreducible characters of degree 3 , we conclude that $(G / K, p, D)=\left(\mathrm{S}_{5}, 3,6\right)$, as stated in (ii).

(b2) $q \equiv \epsilon(\bmod 4)$. In this case, $4 p \geq 12$ divides $q-\epsilon$, so $q \geq 11$. Then $\bar{S}$ has $(q-2+\epsilon) / 4$ irreducible characters of degree $q-\epsilon$, and each of them extends to $\operatorname{PGL}_{2}(q)$. Notice that $\operatorname{Aut}(\bar{S})=\operatorname{PGL}_{2}(q): C_{f}$, so the $\operatorname{Aut}(S)$-orbit of any such $\theta$ 
has length at most $f$. Since $q=r^{f} \geq 11$, we have that $(q-2+\epsilon) / 4>f$, and so $\operatorname{Aut}(\bar{S})$ cannot act transitively on the characters of $\bar{S}$ of degree divisible by $p$.

(b3) $q=2^{f}$ with $f \geq 3$. Then $\bar{S}$ has $(q-1+\epsilon) / 2$ irreducible characters of degree $q-\epsilon$. Notice that $\operatorname{Aut}(\bar{S})=\bar{S}: C_{f}$, so the $\operatorname{Aut}(S)$-orbit of any such $\theta$ has length at most $f$. The transitivity of $\operatorname{Aut}(\bar{S})$ on the set of characters of $\bar{S}$ of degree divisible by $p$ now implies that $f=3, \epsilon=-1, p=3$, and $\bar{S}=\mathrm{SL}_{2}(8) \leq G / K \leq \bar{S}: C_{3}$. But $\mathrm{SL}_{2}(8)$ has 3 irreducible characters of degree 9, and $\operatorname{Aut}\left(\mathrm{SL}_{2}(8)\right)=\bar{S}: C_{3}$ has irreducible characters of both degrees 21 and 27 . This contradiction completes the proof of the proposition.

We now show that the groups satisfying conclusion (i) of Theorem 2.1 have only one irreducible character whose degree is divisible by $p$.

Proposition 2.3. Let $D=q=p^{f} \geq 4$ and assume that the finite group $G$ satisfies conclusion (i) of Theorem 2.1. Then $G$ has exactly one irreducible character $\theta$ of degree divisible by $p$. Furthermore, $\theta(1)=D$.

Proof. (1) The statement is clear if $G=\operatorname{PSL}_{2}(q)$ or $\operatorname{SL}_{2}(q)$. So we consider the third possibility, with $S:=G / V \cong \mathrm{SL}_{2}(q)$ and $|V|=q^{2}$. Note that $S$ has a unique irreducible character of degree divisible by $p$, namely the Steinberg character of $S$, of degree $q$. So it suffices to show that any $\chi \in \operatorname{Irr}(G \mid V)$ has degree $q^{2}-1$.

Fix a nontrivial character $\lambda \in \operatorname{Irr}(V)$. Since $S$ acts transitively on the nonzero vectors of $V$ and $V^{*}$, the stabilizer of $\lambda$ in $G$ is a subgroup $I$ of order $|G| /\left(q^{2}-1\right)=q^{3}$; in particular, $P:=I / V \in \operatorname{Syl}_{p}(S)$. Also we have that $\chi=\mu^{G}$ for some $\mu \in \operatorname{Irr}(I \mid \lambda)$. Thus the map $\mu \mapsto \mu^{G}$ yields a bijection between $\operatorname{Irr}(I \mid \lambda)$ and $\operatorname{Irr}(G \mid V)$.

(2) It suffices to show that there is at least one $\chi \in \operatorname{Irr}(G \mid V)$ of degree $q^{2}-1$. Indeed, if this is the case then there exists $v \in \operatorname{Irr}(I)$ such that $\left.v\right|_{V}=\lambda$. Now by Gallagher's theorem, $\operatorname{Irr}(P \mid \lambda)=\{v \gamma \mid \gamma \in \operatorname{Irr}(I / V)\}$ consists of exactly $q$ characters, all of degree 1 as $P$ is elementary abelian of order $q$. It follows that all characters in $\operatorname{Irr}(G \mid V)$ have degree $q^{2}-1$.

(3) Assume that the extension $G=V S$ is split. Then $I$ splits over $V$ and so $I / \operatorname{Ker}(\lambda)$ is a split extension of $V / \operatorname{Ker}(\lambda) \cong C_{p}$ by $P$. Since $P$ fixes $\lambda, P$ centralizes $V / \operatorname{Ker}(\lambda)$, whence $I / \operatorname{Ker}(\lambda)$ is abelian. Hence all irreducible characters of $I / \operatorname{Ker}(\lambda)$ are of degree 1 and so are all characters in $\operatorname{Irr}(I \mid \lambda)$, whence we are done as in (2).

Suppose now that the extension $G=V S$ is nonsplit. It follows that $H^{2}(S, V) \neq 0$. By a result of McLaughlin (see [Sah 1977, Proposition 4.4]), this can happen only when $p=2$ and $f \geq 3$. In the exceptional case, $H^{2}(S, V)=\mathbb{F}_{q}$, and so there is a unique (up to isomorphism) such nonsplit extension. By [Kostrikin and Tiep 1994, Theorem 1.3.7], this nonsplit extension can be realized as the commutator subgroup of the group of all automorphisms of the standard orthogonal decomposition of the 
complex simple Lie algebra $\mathscr{L}$ of type $A_{q-1}$. Now the action of $G$ on $\mathscr{L}$ gives rise to a faithful irreducible representation of degree $\operatorname{dim}(\mathscr{L})=q^{2}-1$, and so we are done by (2).

In what follows we will keep the notation from conclusion (ii) of Proposition 2.2.

Lemma 2.4. Let $p=3$ and suppose that $G$ is an NP1-group with a normal subgroup $N \cong \mathrm{S}_{5}$ or $M_{11}$. Then $G=N$.

Proof. Certainly $N^{\prime} \triangleleft G$ and $N^{\prime}$ has an irreducible real-valued character $\alpha$ of odd degree $\alpha(1)=3$ or 45. Since $N^{\prime}$ is perfect, $o(\alpha)=1$. By [Navarro and Tiep 2008, Theorem 2.3], $\alpha$ extends to $I:=I_{G}(\alpha)$. Hence, by Gallagher's theorem, there is a bijection between $\operatorname{Irr}(I \mid \alpha)$ and $\operatorname{Irr}\left(I / N^{\prime}\right)$. On the other hand, $\operatorname{Irr}(G \mid \alpha)$ lies in a bijective correspondence with $\operatorname{Irr}(I \mid \alpha)$, and every $\chi \in \operatorname{Irr}(G \mid \alpha)$ has degree divisible by 3. It follows that $\left|\operatorname{Irr}\left(I / N^{\prime}\right)\right|=1$, and so $I=N^{\prime} \leq N$. In particular, $I=I_{N}(\alpha)$. Now observe that $N$, and so $G$, acts transitively on the set $X$ of irreducible characters of $N^{\prime}$ of degree equal to $\alpha(1)$. It follows that $[G: I]=|X|=[N: I]$, whence $G=N$.

In the situation of Proposition 2.2(ii), we set $G_{1}=G$ if $G / K=M_{11}$, and let $G_{1}$ be the unique subgroup of index 2 containing $K$ if $G / K=\mathrm{S}_{5}$ (so that $G_{1} / K=\mathrm{A}_{5}$ ).

Lemma 2.5. Let $G$ be an NP1-group satisfying conclusion (ii) of Proposition 2.2. Let $R \triangleleft G$ be such that $G / R$ is 3-solvable. Then $R \geq G_{1}$.

Proof. Note that $G / K R$ is a 3 -solvable quotient of $G / K=\mathrm{S}_{5}$ or $M_{11}$, hence $K R \geq G_{1}$. Assume first that $K R=G$. Then $G /(K \cap R)$ contains the normal subgroup $R /(K \cap R) \cong G / K$, and $G /(K \cap R)$ is certainly an NP1-group. By Lemma $2.4, R /(K \cap R)=G /(K \cap R)$ and so $R=G$.

We may now assume that $G / K=\mathrm{S}_{5}$ and $K R=G_{1}$. Again $H:=G /(K \cap R)$ is an NP1-group with the normal subgroup $M:=R /(K \cap R) \cong \mathrm{A}_{5}$. Then $H$ has a unique irreducible character $\rho$ of degree divisible by 3 . It follows that $H$ acts transitively on the set $\{\alpha, \beta\}$ of irreducible characters of degree 3 of $M$; in particular, $I:=I_{H}(\alpha)$ has index 2 in $H$. As in the proof of Lemma 2.4, we see that the real character $\alpha$ extends to $I$, and there is a bijection between $\operatorname{Irr}(H \mid \alpha)$ and $\operatorname{Irr}(I / M)$. Since $H$ is an NP1-group, it follows that $I=M$, and so $[G: R]=[H: M]=[H: I]=2$. Recall that $G_{1}=K R \geq R$ and $\left[G: G_{1}\right]=2$. Hence, we must have that $R=G_{1}$.

Lemma 2.6. Let $G$ be an NP1-group satisfying conclusion (ii) of Proposition 2.2. Then $K$ is solvable.

Proof. Assume the contrary: $L / M$ is a nonabelian chief factor of $G$ in $K / U$. Certainly $G / M$ is also an NP1-group. So, modding out by $M$ we may assume that $M=1$ and that $L=T_{1} \times \cdots \times T_{n}$ is a minimal normal subgroup of $G$, with $T_{i} \cong T$, a nonabelian simple $3^{\prime}$-group. Now $G$ acts on the set $\left\{T_{1}, \ldots, T_{n}\right\}$ inducing a transitive subgroup $X$ of $S_{n}$. 
424 D. Goldstein, R. Guralnick, M. Lewis, A. Moretó, G. Navarro and P. H. Tiep

First suppose that $p$ divides $|X|$. By [Casolo and Dolfi 2009, Lemma 8] we can relabel the factors $T_{i}$ and find $1 \leq k \leq k+l \leq n$ such that

$$
\operatorname{Stab}_{X}(\{1,2, \ldots, k\},\{k+1, k+2, \ldots, k+l\})
$$

has index divisible by $p$ in $X$. It follows that

$$
N:=\mathbf{N}_{G}\left(T_{1} \times T_{2} \times \cdots \times T_{k}, T_{k+1} \times T_{k+2} \times \cdots \times T_{k+l}\right)
$$

has index divisible by $p$ in $G$. Also, since $T$ is simple nonabelian, we can find $\alpha, \beta \in \operatorname{Irr}(T)$ of distinct degrees larger than 1 . Consider the irreducible character

$$
\gamma:=(\underbrace{\alpha \times \cdots \times \alpha}_{k}) \times(\underbrace{\beta \times \cdots \times \beta}_{l}) \times(\underbrace{1_{T} \times \cdots \times 1_{T}}_{n-k-l})
$$

of $L$. Then $I_{G}(\gamma) \leq N$ and so has index divisible by $p$ in $G$, contradicting Proposition 2.2.

We have $X$ is a $p^{\prime}$-group. Thus $X=G / Y$ is a $3^{\prime}$-group, if $Y$ denotes the kernel of the action of $G$ on $\left\{T_{1}, \ldots, T_{n}\right\}$. By Lemma $2.5, Y \geq G_{1}$; in particular, $|X| \leq 2$ and so $n \leq 2$. Recall that $T$ is a simple nonabelian $p^{\prime}$-group. Hence the condition $n \leq 2$ now implies that $\operatorname{Aut}(L) \leq \operatorname{Aut}(T) \gtrless \mathrm{S}_{2}$ is 3-solvable, whence $G / \mathbf{C}_{G}(L)$, as a subgroup of $\operatorname{Aut}(T)$, is also 3-solvable. Again by Lemma $2.5, \mathbf{C}_{G}(L) \geq G_{1}$. Since [ $\left.G: G_{1}\right] \leq 2, \mathbf{C}_{G}(L)$ contains $L$ and so $L$ is abelian, a contradiction.

Lemma 2.7. Let $S=M_{11}, p=3$, and let $r$ be a prime. Let $G$ be an NP1-group with a minimal normal $r$-subgroup $V$ such that $G / V=S$. Then $V=1$.

Proof. Assume the contrary: $V \neq 1$. Identify $\operatorname{Irr}(V)$ with the dual module $V^{*}$. By Proposition 2.2, every nonzero $v \in V^{*}$ is fixed by a Sylow 3-subgroup of $S$. We will view $V$ as an absolutely irreducible $\mathbb{E} S$-module of dimension $n$, where $\mathbb{E}:=\operatorname{End}_{S}(V)=\mathbb{F}_{t}$ for some power $t$ of $r$. If $n=1$, then $V \leq \mathbf{Z}(G), V=C_{p}$, and $G \cong V \times S$ (as $S$ has trivial Schur multiplier), contradicting Lemma 2.4. So we will assume $n \geq 2$ and estimate the number $N$ of nonzero elements of $V^{*}$ that are fixed by a Sylow 3-subgroup $P$ of $S$. Let $g \in S$ have order 3. As mentioned in [Guralnick and Saxl 2003], $S$ is generated by three conjugates of $g$. Hence by [Guralnick and Tiep 2005, Lemma 3.2], $g$ can fix at most $t^{\lfloor 2 n / 3\rfloor}$ elements of $V^{*}$. It follows that $N \leq t^{\lfloor 2 n / 3\rfloor}-1$. Also note that $S$ has 55 Sylow 3 -subgroups.

Consider the case $r \neq 3$. Then $n \geq 9$ if $r=11$ and $n \geq 10$ otherwise (see [Jansen et al. 1995]). Since $55 N \geq t^{n}-1=\left|V^{*} \backslash\{0\}\right|$, we must have that $r=2$ and $n \leq 21$. In the remaining cases, using Brauer characters as given in [Jansen et al. 1995], we see that $g$ can fix at most $2^{4}$ elements of $V^{*}$. Thus $N \leq 2^{4}-1<\left(2^{10}-1\right) / 55 \leq$ $\left(t^{n}-1\right) / 55$, again a contradiction.

Now suppose $r=3$; in particular, $n \geq 5$. If $t \geq 9$, or if $t=3$ but $n \geq 10$, then again $55 N<t^{n}-1$, again a contradiction. Thus $t=3$ and $n=5$. Note that $M_{11}$ 
has two irreducible 5-dimensional modules over $\mathbb{F}_{3}$, which are dual to each other. Moreover, the $S$-orbits on them are of lengths 1, 22, and 220, resp. 1, 110, and 132 (see [Liebeck 1987, Table 14]). So $V^{*}$ must be isomorphic to the former module. Direct calculations using [GAP 2004] done by T. Breuer show that in this case the point stabilizer for the vectors in the orbit of length 22 are isomorphic to $A_{6}$ (and isomorphic to $S_{3} \times S_{3}$ for the orbit of length 220). Consider a vector $v$ from the first orbit and view it as $\lambda \in \operatorname{Irr}(V)$, with stabilizer $I$ in $G$. Then $I / \operatorname{Ker}(\lambda) \simeq C_{3} \cdot \mathrm{A}_{6}$, which may be split or nonsplit. In either case, there is $\mu \in \operatorname{Irr}(I \mid \lambda)$ of degree 9 . Now $\mu^{G} \in \operatorname{Irr}(G \mid \lambda)$ has degree $9 \cdot 22=198$, a contradiction (since $G / V=S$ has an irreducible character of degree 45$)$.

Lemma 2.8. Let $S=\mathrm{A}_{5}, p=3$, and let $r$ be a prime. Let $H$ be a finite group with a minimal normal $r$-subgroup $V$ such that $H / V=S$. Assume that the kernel of every irreducible character of degree divisible by 3 of $H$ contains $V$. Then $V=1$.

Proof. Assume the contrary: $V \neq 1$. The condition on $H$ implies by Clifford's theorem that the inertia group of every nontrivial $\lambda \in \operatorname{Irr}(V)$ has $3^{\prime}$-index in $H$. Identifying $\operatorname{Irr}(V)$ with $V^{*}$, we see that every nonzero $v \in V^{*}$ is fixed by a Sylow 3 -subgroup of $S$. View $V$ as an absolutely irreducible $\mathbb{E} S$-module of dimension $n$, where $\mathbb{E}:=\operatorname{End}_{S}(V)=\mathbb{F}_{t}$ for some power $t$ of $r$. If $n=1$, then $V \leq \mathbf{Z}(G), V=C_{3}$, and so $G \cong V \times S$ (as the Schur multiplier of $S$ equals $C_{2}$ ). In this case, $G$ has irreducible characters of degree 3 which are nontrivial at $V$, a contradiction. So we will assume $n \geq 2$. By [Guralnick and Saxl 2003, Lemma 3.1], $S$ is generated by two conjugates of a Sylow 3-subgroup $P$ of $S$. It follows by [Guralnick and Tiep 2005, Lemma 3.2] that $P$ can fix at most $t^{\lfloor n / 2\rfloor}$ elements of $V^{*}$. Since $S$ has 10 Sylow 3-subgroups, we must have

$$
10\left(t^{\lfloor n / 2\rfloor}-1\right) \geq\left|V^{*} \backslash\{0\}\right|=t^{n}-1 ;
$$

in particular, $t^{n} \leq 81$.

Now if $r \geq 5$, then $n \geq 3$, a contradiction. Suppose $r=3$. Then $n=3$ or 4 . In the former case, $t \geq 9$ (see [Jansen et al. 1995]), a contradiction. So $n=4$ and $t=3$. We can now realize $V^{*}$ as the deleted permutation module

$$
\mathbb{F}_{3}^{4}=\left\{\sum_{i=1}^{5} a_{i} e_{i} \mid a_{i} \in \mathbb{F}_{3}, \sum_{i=1}^{5} a_{i}=0\right\}
$$

for $\mathrm{A}_{5}$. But in this case the $\mathrm{A}_{5}$-orbit of $v:=e_{1}+e_{2}-e_{3}-e_{4} \in V^{*}$ has length 30 , a contradiction.

Suppose now $r=2$. If $n=2$, then $P$ has no nonzero fixed points on $V^{*}$, a contradiction. Thus $n=4, t=2$. In this case, either $H \cong V \times \mathrm{A}_{5}$ or $H$ is perfect. In the former case, $H$ has irreducible characters of degree 3 which are nontrivial at $V$, 
a contradiction. In the latter case, using [GAP 2004] one can check that $H$ has irreducible characters of degree 15 which are nontrivial at $V$, again a contradiction.

Proposition 2.9. Let $G$ be an NP1-group satisfying conclusion (ii) of Proposition 2.2. Then $G$ satisfies conclusion (ii) of Theorem 2.1. Conversely, if $G=\mathrm{S}_{5}$ or $M_{11}$, then $G$ is an NP1-group.

Proof. Suppose $G$ satisfies Proposition 2.2(ii). By Proposition 2.2 and Lemma 2.6, $K$ is solvable. Let $G$ be a minimal counterexample, so that $K \neq 1$. By minimality, $K$ is a minimal normal $r$-subgroup for some prime $r$. By Lemma $2.7, G / K=\mathrm{S}_{5}$. Since $G / K$ already has an irreducible character of degree 6 , every irreducible character of $G$ of degree divisible by 3 must be nontrivial at $K$. The same is true for the normal subgroup $G_{1}$ of $G$ (recall $G_{1} / K \cong \mathrm{A}_{5}$ ). Modding out by a suitable normal subgroup inside $K$, we may assume that $K$ is a minimal normal subgroup of $G_{1}$. Now we can apply Lemma 2.8 to get a contradiction. The converse statement is obvious.

We have completed the proof of Theorem 2.1 and of the main theorem.

\section{References}

[Casolo and Dolfi 2009] C. Casolo and S. Dolfi, "Products of primes in conjugacy class sizes and irreducible character degrees", Israel J. Math. 174 (2009), 403-418. MR 2011e:20039 Zbl 1191.20024

[Chillag and Macdonald 1984] D. Chillag and I. D. Macdonald, "Generalized Frobenius groups", Israel J. Math. 47:2-3 (1984), 111-122. MR 85h:20035 Zbl 0533.20012

[Dolfi et al. 2009] S. Dolfi, A. Moretó, and G. Navarro, "The groups with exactly one class of size a multiple of p", J. Group Theory 12:2 (2009), 219-234. MR 2010b:20031 Zbl 1166.20018

[Dolfi et al. 2013] S. Dolfi, G. Navarro, and P. H. Tiep, "Finite groups whose same degree characters are Galois conjugate", Israel J. Math. 198:1 (2013), 283-331. MR 3096641

[Feit 1993] W. Feit, "Extending Steinberg characters", pp. 1-9 in Linear algebraic groups and their representations (Los Angeles, 1992), edited by R. S. Elman et al., Contemp. Math. 153, Amer. Math. Soc., Providence, RI, 1993. MR 94k:20012 Zbl 0823.20013

[Fernández-Alcober and Moretó 2001] G. A. Fernández-Alcober and A. Moretó, "Groups with two extreme character degrees and their normal subgroups", Trans. Amer. Math. Soc. 353:6 (2001), 2171-2192. MR 2002a:20007 Zbl 0968.20005

[Gagola 1983] S. M. Gagola, Jr., "Characters vanishing on all but two conjugacy classes", Pacific J. Math. 109:2 (1983), 363-385. MR 85e:20009 Zbl 0536.20005

[GAP 2004] The GAP group, GAP_-groups, algorithms, and programming, version 4.4, 2004, Available at http://www.gap-system.org.

[Guralnick and Saxl 2003] R. M. Guralnick and J. Saxl, "Generation of finite almost simple groups by conjugates”, J. Algebra 268:2 (2003), 519-571. MR 2005f:20057 Zbl 1037.20016

[Guralnick and Tiep 2005] R. M. Guralnick and P. H. Tiep, "The non-coprime $k(G V)$ problem", $J$. Algebra 293: 1 (2005), 185-242. MR 2006g:20018 Zbl 1083.20006

[Huppert 1957] B. Huppert, “Zweifach transitive, auflösbare Permutationsgruppen”, Math. Z. 68 (1957), 126-150. MR 20 \#904 Zbl 0079.25502 
[Huppert and Blackburn 1982] B. Huppert and N. Blackburn, Finite groups, III, Grundlehren der Mathematischen Wissenschaften [Fundamental Principles of Mathematical Sciences] 243, Springer, Berlin, 1982. MR 84i:20001b Zbl 0514.20002

[Isaacs 1976] I. M. Isaacs, Character theory of finite groups, Pure and Applied Mathematics 69, Academic Press, New York, 1976. MR 57 \#417 Zbl 0337.20005

[Isaacs 1989] I. M. Isaacs, "Coprime group actions fixing all nonlinear irreducible characters", Canad. J. Math. 41:1 (1989), 68-82. MR 90j:20038 Zbl 0686.20002

[Isaacs 2011] I. M. Isaacs, "Bounding the order of a group with a large character degree", J. Algebra 348 (2011), 264-275. MR 2012j:20027 Zbl 1242.20011

[Isaacs et al. 2009] I. M. Isaacs, A. Moretó, G. Navarro, and P. H. Tiep, "Groups with just one character degree divisible by a given prime”, Trans. Amer. Math. Soc. 361:12 (2009), 6521-6547. MR 2010i:20008 Zbl 1203.20005

[Jansen et al. 1995] C. Jansen, K. Lux, R. Parker, and R. Wilson, An atlas of Brauer characters, London Mathematical Society Monographs. New Series 11, Oxford University Press, New York, 1995. MR 96k:20016 Zbl 0831.20001

[Kostrikin and Tiep 1994] A. I. Kostrikin and P. H. Tiep, Orthogonal decompositions and integral lattices, Expositions in Mathematics 15, de Gruyter, Berlin, 1994. MR 96f:17001 Zbl 0855.11033

[Lewis 2001] M. L. Lewis, "Bounding Fitting heights of character degree graphs", J. Algebra 242:2 (2001), 810-818. MR 2003d:20010 Zbl 0989.20007

[Lewis and White 2003] M. L. Lewis and D. L. White, "Connectedness of degree graphs of nonsolvable groups”, J. Algebra 266:1 (2003), 51-76. MR 2004g:20008

[Lewis et al. 2005] M. L. Lewis, A. Moretó, and T. R. Wolf, "Non-divisibility among character degrees”, J. Group Theory 8:5 (2005), 561-588. MR 2006j:20012 Zbl 1097.20011

[Liebeck 1987] M. W. Liebeck, "The affine permutation groups of rank three", Proc. London Math. Soc. (3) 54:3 (1987), 477-516. MR 88m:20004 Zbl 0621.20001

[Manz and Wolf 1993] O. Manz and T. R. Wolf, Representations of solvable groups, London Mathematical Society Lecture Note Series 185, Cambridge University Press, 1993. MR 95c:20013 Zbl 0928.20008

[Manz et al. 1988] O. Manz, R. Staszewski, and W. Willems, "On the number of components of a graph related to character degrees”, Proc. Amer. Math. Soc. 103:1 (1988), 31-37. MR 89b:20030 Zbl 0645.20005

[McKay 1980] J. McKay, "Graphs, singularities, and finite groups", pp. 183-186 in The Santa Cruz Conference on Finite Groups (Santa Cruz, CA, 1979), edited by B. Cooperstein and G. Mason, Proc. Sympos. Pure Math. 37, Amer. Math. Soc., Providence, R.I., 1980. MR 82e:20014 Zbl 0451.05026

[Navarro and Tiep 2008] G. Navarro and P. H. Tiep, "Rational irreducible characters and rational conjugacy classes in finite groups”, Trans. Amer. Math. Soc. 360:5 (2008), 2443-2465. MR 2008k:20014 Zbl 1137.20009

[Noritzsch 1995] T. Noritzsch, "Groups having three complex irreducible character degrees", $J$. Algebra 175:3 (1995), 767-798. MR 96d:20010 Zbl 0839.20014

[Passman 1968] D. Passman, Permutation groups, W. A. Benjamin, New York and Amsterdam, 1968. MR 38 \#5908 Zbl 0179.04405

[Sah 1977] C. H. Sah, “Cohomology of split group extensions, II”, J. Algebra 45:1 (1977), 17-68. MR 57 \#3217 Zbl 0352.18021

[Seitz 1968] G. Seitz, "Finite groups having only one irreducible representation of degree greater than one”, Proc. Amer. Math. Soc. 19 (1968), 459-461. MR 36 \#5212 Zbl 0244.20010 
428 D. Goldstein, R. Guralnick, M. Lewis, A. Moretó, G. Navarro and P. H. Tiep

[Steinberg 1985] R. Steinberg, "Finite subgroups of $\mathrm{SU}_{2}$, Dynkin diagrams and affine Coxeter elements", Pacific J. Math. 118:2 (1985), 587-598. MR 86g:20016 Zbl 0567.20026

Communicated by David Benson

Received 2013-02-20 Revised 2013-05-06 Accepted 2013-07-11

danielgolds@gmail.com

Center for Communications Research, 4320 Westerra Court, San Diego, CA 92121, United States

guralnic@usc.edu

Department of Mathematics, University of Southern California, 3620 S. Vermont Ave., Los Angeles, CA 90089, United States

lewis@math.kent.edu

Department of Mathematical Sciences, Kent State University, Kent, $\mathrm{OH}$ 44242, United States

alexander.moreto@uv.es

Departament dÁlgebra, Facultat de Matemátiques, Universitat de Valéncia, Burjassot, 46100 Valéncia, Spain

gabriel.navarro@uv.es

Departament dÁlgebra, Facultat de Matemátiques, Universitat de Valéncia, Burjassot, 46100 Valéncia, Spain

tiep@math.arizona.edu

Department of Mathematics, University of Arizona, 617 N. Santa Rita Ave., P.O. Box 210089,

Tucson, AZ 85721-0089, United States 


\section{Algebra \& Number Theory}

msp.org/ant

\section{EDITORS}

MANAGING EDITOR

Bjorn Poonen

Massachusetts Institute of Technology

Cambridge, USA

\author{
EDITORIAL BOARD CHAIR \\ David Eisenbud \\ University of California \\ Berkeley, USA
}

\section{BOARD OF EDITORS}

Georgia Benkart

Dave Benson

Richard E. Borcherds

John H. Coates

J-L. Colliot-Thélène

Brian D. Conrad

Hélène Esnault

Hubert Flenner

Edward Frenkel

Andrew Granville

Joseph Gubeladze

Roger Heath-Brown

Ehud Hrushovski

Craig Huneke

Mikhail Kapranov

Yujiro Kawamata

János Kollár

Yuri Manin

Barry Mazur

Philippe Michel
University of Wisconsin, Madison, USA

University of Aberdeen, Scotland

University of California, Berkeley, USA

University of Cambridge, UK

CNRS, Université Paris-Sud, France

University of Michigan, USA

Freie Universität Berlin, Germany

Ruhr-Universität, Germany

University of California, Berkeley, USA

Université de Montréal, Canada

San Francisco State University, USA

Oxford University, UK

Hebrew University, Israel

University of Virginia, USA

Yale University, USA

University of Tokyo, Japan

Princeton University, USA

Northwestern University, USA

Harvard University, USA

École Polytechnique Fédérale de Lausanne
Susan Montgomery

Shigefumi Mori

Raman Parimala

Jonathan Pila

Victor Reiner

Karl Rubin

Peter Sarnak

Joseph H. Silverman

Michael Singer

Vasudevan Srinivas

J. Toby Stafford

Bernd Sturmfels

Richard Taylor

Ravi Vakil

Michel van den Bergh

Marie-France Vignéras

Kei-Ichi Watanabe

Efim Zelmanov

Shou-Wu Zhang
University of Southern California, USA

RIMS, Kyoto University, Japan

Emory University, USA

University of Oxford, UK

University of Minnesota, USA

University of California, Irvine, USA

Princeton University, USA

Brown University, USA

North Carolina State University, USA

Tata Inst. of Fund. Research, India

University of Michigan, USA

University of California, Berkeley, USA

Harvard University, USA

Stanford University, USA

Hasselt University, Belgium

Université Paris VII, France

Nihon University, Japan

University of California, San Diego, USA

Princeton University, USA

PRODUCTION

production@msp.org

Silvio Levy, Scientific Editor

See inside back cover or msp.org/ant for submission instructions.

The subscription price for 2014 is US $\$ 225 /$ year for the electronic version, and $\$ 400 /$ year $(+\$ 55$, if shipping outside the US) for print and electronic. Subscriptions, requests for back issues and changes of subscribers address should be sent to MSP.

Algebra \& Number Theory (ISSN 1944-7833 electronic, 1937-0652 printed) at Mathematical Sciences Publishers, 798 Evans Hall \#3840, c/o University of California, Berkeley, CA 94720-3840 is published continuously online. Periodical rate postage paid at Berkeley, CA 94704, and additional mailing offices.

ANT peer review and production are managed by EditFLOW ${ }^{\circledR}$ from Mathematical Sciences Publishers.

\section{PUBLISHED BY}

- mathematical sciences publishers

nonprofit scientific publishing

http://msp.org/

(C) 2014 Mathematical Sciences Publishers 


\section{Algebra \& Number Theory}

Volume $8 \quad$ No. $2 \quad 2014$

Large self-injective rings and the generating hypothesis

LEIGH SHEPPERSON and NEIL STRICKLAND

On lower ramification subgroups and canonical subgroups

303

SHIN HATTORI

Wild models of curves

DINO LORENZINI

Geometry of Wachspress surfaces

COREY IRVING and HAL SCHENCK

Daniel Goldstein, Robert M. GuRAlnick, Mark L. Lewis, AleXander Moretó, Gabriel Navarro and Pham HuU TieP

The homotopy category of injectives

AMNON NEEMAN

Essential dimension of spinor and Clifford groups

Vladimir Chernousov and AleXANDER MERKurJeV

On Deligne's category $\underline{\operatorname{Rep}}^{a b}\left(S_{d}\right)$

JONATHAN COMES and VICTOR OSTRIK

Algebraicity of the zeta function associated to a matrix over a free group algebra

CHRISTIAN KASSEL and Christophe REUTENAUER 